\title{
Multi-messenger tests of cosmic-ray acceleration in radiatively inefficient accretion flows
}

\author{
Shigeo S. Kimura ${ }^{1,2,3,4,5}$, Kohta Murase ${ }^{1,2,3,6}$, and Peter Mészáros ${ }^{1,2,3}$ \\ ${ }^{1}$ Department of Physics, The Pennsylvania State University, University Park, Pennsylvania 16802, USA \\ ${ }^{2}$ Department of Astronomy \& Astrophysics, The Pennsylvania State University, University Park, Pennsylvania 16802, USA \\ ${ }^{3}$ Center for Particle and Gravitational Astrophysics, \\ The Pennsylvania State University, University Park, Pennsylvania 16802, USA \\ ${ }^{4}$ Frontier Research Institute for Interdisciplinary Sciences, Tohoku University, Sendai 980-8578, Japan \\ ${ }^{5}$ Astronomical Institute, Tohoku University, Sendai 980-8578, Japan and \\ ${ }^{6}$ Yukawa Institute for Theoretical Physics, Kyoto, Kyoto 606-8502 Japan
}

(Dated: December 3, 2019)

\begin{abstract}
The cores of active galactic nuclei (AGNs) have been suggested as the sources of IceCube neutrinos, and recent numerical simulations have indicated that hot AGN coronae of Seyfert galaxies and radiatively inefficient accretion flows (RIAFs) of low-luminosity AGNs (LLAGNs) may be promising sites of ion acceleration. We present detailed studies on detection prospects of high-energy multimessenger emissions from RIAFs in nearby LLAGNs. We construct a model of RIAFs that can reproduce the observational features of the current X-ray observations of nearby LLAGNs. We then calculate the high-energy particle emissions from nearby individual LLAGNs, including MeV gamma rays from thermal electrons, $\mathrm{TeV}-\mathrm{PeV}$ neutrinos produced by non-thermal protons, and sub-GeV to sub-TeV gamma rays from proton-induced electromagnetic cascades. We find that, although these are beyond the reach of current facilities, proposed future experiments such as e-ASTROGAM and IceCube-Gen2 should be able to detect the $\mathrm{MeV}$ gamma rays and the neutrinos, respectively, or else they can place meaningful constraints on the parameter space of the model. On the other hand, the detection of high-energy gamma rays due to the electromagnetic cascades will be challenging with the current and near-future experiments, such as Fermi and Cherenkov Telescope Array. In an accompanying paper, we demonstrate that LLAGNs can be a source of the diffuse soft gamma-ray and $\mathrm{TeV}-\mathrm{PeV}$ neutrino backgrounds, whereas in the present paper, we focus on the prospects for multi-messenger tests which can be applied to reveal the nature of the high-energy neutrinos and photons from LLAGNs.
\end{abstract}

\section{INTRODUCTION}

The IceCube Collaboration reported the detection of extraterrestrial neutrinos in 2013 [1, 2] and provided the details of the diffuse neutrino background intensity 3 [5]. The origin of the astrophysical neutrino background has yet to be confirmed (see Ref. [6] for a review). Gammaray bursts (GRBs) were expected to be a promising neutrino source 7 11]. However, stacking analyses using the information of the observed GRBs detect no associated neutrinos, which puts an upper limit on the GRB contribution to the neutrino intensity of $\lesssim 1 \%, 12,13]$. Note that these analyses focus on the prompt emission from bright GRBs, while neutrinos produced in the afterglow phase 14 17], by low-luminosity GRBs and enginedriven supernovae 18 22], or by failed GRBs (also known as choked jets; 8, 23-29]) are not very constrained.

Blazars are also believed to be capable of strong neutrino emission [30 32]. Recently, IceCube reported the detection of a high-energy neutrino coincident with a flaring activity of a blazar, TXS 0506+056 33. Thanks to the ensuing multi-messenger followup campaign (see [34]), the broad-band spectral energy distribution during the flaring period has been determined, which enables one to model the neutrino emission in detail [35 39]. The IceCube Collaboration also found a neutrino flare from this object during 2014 - 2015, by re-analyzing their archival data [40]. However, this neutrino flare is not accompa- nied by a corresponding $\mathrm{GeV}$ gamma-ray flaring activity 41], which challenges the theoretical modeling of the neutrino emission [36, 42 44]. Note that the coincident detection and the archival neutrino flare do not, however, mean that the blazars are the dominant source of the diffuse neutrinos. The stacking analyses of the blazars detected by Fermi result in a non-detection 45-48], which implies that their contribution is less than $\sim 10-30 \%$ of the total astrophysical neutrinos. Also, the absence of event clustering in the arrival distribution of neutrinos indicates that the contributions from flaring blazars should be less than $\sim 10-50 \%$ [36, 49].

Another constraint is provided by the extragalactic gamma-ray background detected by Fermi [50]. When astrophysical neutrinos are produced through pion decay, gamma rays are also produced simultaneously. The generated gamma-ray luminosity is comparable to the neutrino luminosity, and the $\mathrm{TeV}-\mathrm{PeV}$ gamma rays are cascaded down to the $\mathrm{GeV}-\mathrm{TeV}$ energy range during their propagation towards Earth. In order to avoid overproducing the observed extragalactic gamma-ray background, the neutrino spectral index should be smaller than $2.1-2.2$ [51], which is in tension with the best-fit spectrum of the observed neutrinos in the shower analyses [4, 5, 52, 53]. Also, the neutrino flux at 1-100 TeV is higher than that above $100 \mathrm{TeV}$ [4, 5], although this might be due to the strong atmospheric background [54]. If such a "medium-energy excess" is real, the serious ten- 
sion with the gamma-ray background is unavoidable, suggesting that the main sources are opaque and hidden in high-energy gamma rays 55]. This argument disfavors many astrophysical scenarios as the origin of these neutrinos, including starburst galaxies [51, 56 64], galaxy clusters [51, 65 69], and radio-galaxies [70, 71].

We consider high-energy neutrino emission from the vicinity of supermassive black holes (SMBHs) in active galactic nuclei (AGNs) 72 78]. A luminous AGN hosts a geometrically thin, optically thick accretion disk that produces copious UV photons 79 81, and the ratio of the observed UV to X-ray luminosity is very high 82 84. Such target photon fields lead to a hard neutrino spectrum at PeV energies [85, 86]. The accretion shock has been considered, but the existence of such a shock has not been supported by numerical simulations so far. On the other hand, recent studies on magnetorotational instabilities suggest that particle acceleration via magnetic reconnections and turbulence is promising in AGN coronae, and Ref. 87] showed that the mysterious $10-$ $100 \mathrm{TeV}$ component in the diffuse neutrino flux can be explained by the AGN core model of radio-quiet AGNs. It was found that the Bethe-Heitler process is critically important, which led to robust predictions of $\mathrm{MeV}$ gamma rays via proton-induced cascades.

Low-luminosity AGNs (LLAGNs), however, have different spectral energy distributions, in which an UV bump is absent [88]. This indicates that there is an optically thin, hot accretion flow instead of an optically thick disk. Remarkably, plasma properties of hot AGN coronae and radiatively inefficient accretion flows (RIAF; 89, 90]) in LLAGNs seem similar in the sense that the plasmas are expected to be collisionless for ions. It is natural to consider the same type of proton acceleration in both Seyfert galaxies and LLAGNs. Ref. 91] considered the stochastic acceleration expected in such RIAFs of LLAGNs, and showed that the neutrinos produced by the accelerated protons can account for the diffuse astrophysical neutrino background (see also Refs. [92, 93] for neutrino emissions from LLAGNs). The LLAGN model can avoid the gamma-ray and the point-source constraints, thanks to its compact emission region and high number density, although Ref. 91] did not provide details of the resulting gamma-ray spectra.

In this paper, we describe a refined LLAGN model, and show how multi-messenger information on neutrinos and gamma rays can be used as a test of the proposed LLAGN model. We estimate the physical quantities in the RIAFs of several nearby LLAGNs including the photons from the thermal electrons in Section II] We then estimate the high-energy proton spectra in Section III. and calculate the high-energy neutrino spectra and their detectability in Section IV. We calculate the gamma rays from proton-induced electromagnetic cascades in Section V] Finally, we summarize the results and discuss their implications in Section VI. We note that our refined model can reproduce the diffuse $\mathrm{MeV}$ gamma-ray and the $\mathrm{TeV}-$ PeV neutrino backgrounds simultaneously without over- shooting the Fermi data, which is shown in an accompanying paper. In this paper, we focus on the detection prospects of individual nearby LLAGNs.

\section{PHYSICAL QUANTITIES IN RIAFS}

We consider a RIAF of size $R$ and mass accretion rate $\dot{M}$ around a SMBH of mass $M_{\mathrm{BH}}$. We use the notation $Q_{x}=10^{x}$ in cgs units, unless otherwise noted. To represent the physical quantities in the RIAF, it is convenient to normalize $R$ by the Schwarzschild radius: $R=\mathcal{R} R_{S} \simeq 2.95 \times 10^{14} \mathcal{R}_{1} M_{8}$, where $R_{S}=2 G M_{\mathrm{BH}} / c^{2}$ is the Schwarzschild radius, $G$ is the gravitational constant, and $c$ is the speed of light. The mass accretion rate is normalized by the Eddington accretion rate: $\dot{m}=\dot{M} c^{2} / L_{\mathrm{Edd}}$, where $L_{\mathrm{Edd}} \simeq 1.3 \times 10^{46} M_{8} \mathrm{erg} \mathrm{s}^{-1}$ is the Eddington luminosity.

According to recent magnetohydrodynamic (MHD) simulations (see e.g., Refs. 94 99]), the radial velocity, the sound velocity, the scale height, the number density, the magnetic field, and the Alfven velocity in the RIAF are estimated to be

$$
\begin{aligned}
V_{R} & \approx \frac{1}{2} \alpha V_{K} \simeq 3.4 \times 10^{8} \mathcal{R}_{1}^{-1 / 2} \alpha_{-1} \mathrm{~cm} \mathrm{~s}^{-1} \\
C_{s} & \approx \frac{1}{2} V_{K} \simeq 3.4 \times 10^{9} \mathcal{R}_{1}^{-1 / 2} \mathrm{~cm} \mathrm{~s}^{-1} \\
H & \approx \frac{1}{2} R \simeq 1.5 \times 10^{14} \mathcal{R}_{1} M_{8} \mathrm{~cm} \\
n_{p} & \approx \frac{\dot{M}}{4 \pi m_{p} R H V_{R}} \simeq 4.6 \times 10^{8} \mathcal{R}_{1}^{-3 / 2} \alpha_{-1}^{-1} M_{8}^{-1} \dot{m}_{-2} \mathrm{~cm}^{-3} \\
B & \approx \sqrt{\frac{8 \pi P_{g}}{\beta}} \simeq 2.6 \times 10^{2} \mathcal{R}_{1}^{-5 / 4} \alpha_{-1}^{-1 / 2} M_{8}^{-1 / 2} \dot{m}_{-2}^{1 / 2} \beta_{0.5}^{-1 / 2} \mathrm{G} \\
V_{A} & \approx \frac{B}{\sqrt{4 \pi m_{p} n_{p}}} \simeq 2.7 \times 10^{9} \mathcal{R}_{1}^{-1 / 2} \beta_{0.5}^{-1 / 2} \mathrm{~cm} \mathrm{~s}^{-1}
\end{aligned}
$$

where $V_{K}=\sqrt{G M_{\mathrm{BH}} / R}$ is the Keplerian velocity, $\alpha$ is the viscous parameter [79], $m_{p}$ is the proton mass, $\beta=8 \pi P_{g} / B^{2}$ is the plasma beta, and $P_{g}=m_{p} n_{p} C_{s}^{2}$ is the gas pressure. We assume pure proton composition for simplicity. The magnetic field strength in the hot accretion flows depends on the configuration of the magnetic field: $\beta \sim 10-100$ for standard and normal evolution (SANE) flows, whereas $\beta \sim 1-10$ for magnetically arrested disks (e.g., [95, 97, 100, 101]). We use $\beta \sim 3.2$ as a reference value because lower $\beta$ plasma are suitable for producing non-thermal particles 102]. For the viscous parameter $\alpha$, SANE models tend to give a lower value, $\alpha \simeq 0.03$ [98, 99], while observations of X-ray binaries and dwarf novae suggest $\alpha \simeq 0.1-1$ (see Ref. [103] and references therein). Here, we set $\alpha=0.1$ as a reference value.

Although cooling processes have little influence on the dynamical structure in the RIAF, the thermal electrons supply target photons for photohadronic interactions and $\gamma \gamma$ two-photon annihilation. We calculate the 
characteristics of the target photons in the RIAF using a method similar to Ref. [91]. We consider synchrotron, bremsstrahlung, and inverse Compton emission processes. The calculation method of the emission spectrum due to each process was discussed in the Appendix of Ref. [91]. Note that this treatment is valid only for flows with Thomson optical depths $\tau_{T} \approx n_{p} \sigma_{T} R<1$, where $\sigma_{T}$ is the Thomson cross section.

As long as $\dot{m} \gtrsim 10^{-2} \alpha^{2} \sim 10^{-4} \alpha_{-1}^{2}$, the balance between the cooling rate and heating rate of the thermal electrons determines the electron temperature, $\Theta_{e}=$ $k_{B} T_{e} /\left(m_{e} c^{2}\right)$, where $m_{e}$ is the electron mass and $k_{B}$ is the Boltzmann constant [90, 104]. Then, the electron heating rate is equal to the bolometric luminosity from the thermal electrons. If the Coulomb collisions with the thermal protons are the dominant heating process, the heating rate is proportional to $n_{p}^{2}$, which leads to $L_{\mathrm{bol}} \propto \dot{m}^{2}$. Then, the bolometric luminosity is phenomenologically given by (see, e.g., Ref. [105, 106])

$$
L_{\mathrm{bol}} \approx \epsilon_{\mathrm{rad}, \mathrm{sd}}\left(\frac{\dot{m}}{\dot{m}_{\mathrm{crit}}}\right)^{2} \dot{m}_{\mathrm{crit}} L_{\mathrm{Edd}},
$$

where $\dot{m}_{\text {crit }}$ is the normalized critical accretion rate above which the RIAF solution no longer exists [107, 108] and $\epsilon_{\mathrm{rad}, \mathrm{sd}} \sim 0.1$ is the radiation efficiency of the standard thin disk. The critical accretion rate can be expressed as a function of $\alpha$ [104, 109]. Following Ref. [109], we represent $\dot{m}_{\text {crit }} \sim 3 \alpha^{2} \simeq 3 \times 10^{-2} \alpha_{-1}^{2}$. Note that the dissipation processes in collisionless accretion flows are still controversial. If the electrons are directly heated by plasma dissipation processes induced by kinetic instabilities [110 118], the electron heating rate may be proportional to $\dot{m}$, leading to $L_{\mathrm{bol}} \propto \dot{m}$ as assumed in Ref. 91]. In reality, the scaling relation may be located between the two regimes. In this paper, we use Equation (11) for simplicity.

Observations give us the X-ray luminosity, $L_{X}$, which is connected to $\dot{m}$ in our model. Using the bolometric correction factor, $\kappa_{\mathrm{bol} / \mathrm{X}}$, the $\mathrm{X}$-ray luminosity is related to the bolometric luminosity as

$$
L_{\mathrm{bol}} \approx \kappa_{\mathrm{bol} / X} L_{X}
$$

According to the $\mathrm{X}$-ray surveys, $\kappa_{\mathrm{bol} / X}$ is higher for a higher $L_{\mathrm{bol}}$ or $\lambda_{\mathrm{Edd}}$, where $\lambda_{\mathrm{Edd}}=L_{\mathrm{bol}} / L_{\mathrm{Edd}}$ is the Eddington ratio. At the low-luminosity end, $\kappa_{\mathrm{bol} / X}$ becomes almost constant, $\kappa_{\mathrm{bol} / X} \sim 5-20$ [84, 119, [120]. Using Equations (11) and (2) with a constant $\kappa_{\mathrm{bol} / X}$, we can write $\dot{m}$ as a function of observables:

$\dot{m} \approx\left(\frac{\kappa_{\mathrm{bol} / X} L_{X} \dot{m}_{\mathrm{crit}}}{\epsilon_{\mathrm{rad}, \mathrm{sd}} L_{\mathrm{Edd}}}\right)^{1 / 2} \approx 1.9 \times 10^{-2} M_{8}^{-1 / 2} L_{X, 42}^{1 / 2} \alpha_{-1}$,

where we use $\kappa_{\mathrm{bol} / X}=15$ and $\epsilon_{\mathrm{rad}, \mathrm{sd}}=0.1$. This $\dot{m}$ is less than $\dot{m}_{\text {crit }}$. Hence, typical LLAGNs with $L_{X} \lesssim$ $10^{42} \mathrm{erg} \mathrm{s}^{-1}$ can host RIAFs.

We calculate spectral energy distributions of nearby LLAGNs listed in Table A.3 of Ref. [121], which pro- vides $M_{\mathrm{BH}}, L_{X}$, luminosity distance $\left(d_{L}\right)$, and declination angle $(\delta)$ for 70 LLAGNs. The mass accretion rate of the listed LLAGNs is estimated using Equation (3) with $\kappa_{\mathrm{bol} / X}=15$. We find that 7 of them have standard disks, i.e., $\dot{m}>\dot{m}_{\text {crit }}$, while the others host RIAFs. Figure 1 shows the target photon spectra from 4 LLAGNs whose parameters and resulting physical quantities are tabulated in Table $\square$ and $[1$ respectively. The values of the other parameters are tabulated in Table III The four LLAGNs differ in $M_{\mathrm{BH}}$ and $\dot{m}$. NGC 3516, NGC 4203, and NGC 5866 have $M_{\mathrm{BH}}$ close to $10^{8} \mathrm{M}_{\odot}$, while NGC 3998 hosts a SMBH of $M_{\mathrm{BH}} \sim 10^{9} \mathrm{M}_{\odot} . \dot{m}$ is close to the critical accretion rate for NGC 3516, $\dot{m} \sim 0.1 \dot{m}_{\text {crit }}$ for NGC 4203 and NGC 3998, and $\dot{m} \sim 0.01 \dot{m}_{\text {crit }}$ for NGC 5866. For all the LLAGNs, the synchrotron emission peaks in the radio band. For LLAGNs with $\dot{m} \gtrsim 10^{-2} \dot{m}_{\text {crit }}$, the inverse Compton emission of the synchrotron photons produces infrared to $\mathrm{MeV}$ photons. For lower $\dot{m}$ cases, the bremsstrahlung emits $\mathrm{MeV}$ photons due to inefficient Comptonization. The inverse Compton emission spectrum is hard and smooth for higher $\dot{m}$, while it is soft and bumpy for lower $\dot{m}$ due to a high value of electron temperature and a low value of Compton- $Y$ parameter, $y \approx \tau_{T}\left(4 \Theta_{e}+16 \Theta_{e}^{2}\right)$ (see Table $\rrbracket$ for the values of $\dot{m}, \Theta_{e}$, and $\tau_{T}$ ). A high value of $M_{\mathrm{BH}}$ with fixed $\dot{m}$ lowers the peak frequency of the synchrotron emission due to the weak magnetic field, and increases the entire luminosity because of a high net accretion luminosity, $\dot{M} c^{2}=\dot{m} L_{\mathrm{Edd}} \propto M_{\mathrm{BH}}$.

Next, we compare the X-ray luminosities obtained by our calculations and observations. Figure 2 shows the relation between the observed $2-10 \mathrm{keV}$ X-ray luminosity, $L_{X \text {,obs }}$, and the X-ray luminosity calculated by our model, $L_{X, \text { calc }}$ in the same band. Intriguingly, our simple model is in a good agreement with the observations for $\dot{m}>10^{-3}$. The two luminosities match within a factor of 1.7 in this sample. We stress that we do not adjust the X-ray luminosity but we calculate photon spectra with the one-zone model using $\dot{m}$ estimated by Equations (2) and (3). For a lower value of $\dot{m}<10^{-3}$, the synchrotron emission is more efficient than the inverse Compton emission. This causes a higher value of $\kappa_{\text {bol } / X}$, resulting in $L_{X \text {,calc }}<L_{X \text {,obs }}$ as seen in the figure. For nearby low-ionization nuclear emission-like regions (LINERs), the bolometric correction factor is estimated to be $\kappa_{\mathrm{bol} / X} \sim 50$ [122]. $L_{X \text {,calc }}$ is higher with such a higher value of $\kappa_{\mathrm{bol} / X}$, since it leads to a higher value of $\dot{m}$. Hence, a higher value of $\kappa_{\mathrm{bol} / X}$ is more consistent with our model with $\dot{m}<10^{-3}$. Nevertheless, we use $\kappa_{\text {bol } / X}=15$ because LLAGNs with $\dot{m}<10^{-3}$ do not affect the detectability of high-energy neutrinos as shown in Section IV

The bright LLAGNs are detected by the Swift BAT, most of which show hard X-ray spectra. Thus, very interestingly, our model is consistent with the BAT data in terms of luminosity. In addition, RIAF models generally predict that a higher $\dot{m}$ object has a harder photon spectrum in the X-ray band owing to a higher value of the 
TABLE I. Observational quantities for nearby LLAGNs. These LLAGNs are selected as the ten brightest ones in Xray band except NGC 5866, which is an LLAGN with a lower accretion rate shown in Figure [1. Units are $\left[\mathrm{erg} \mathrm{s}^{-1} \mathrm{~cm}^{-2}\right]$ for $F_{X, \mathrm{obs}},\left[\mathrm{erg} \mathrm{s}^{-1}\right]$ for $L_{X, \mathrm{obs}},\left[\mathrm{M}_{\odot}\right]$ for $M_{\mathrm{BH}},[\mathrm{Mpc}]$ for $d_{L}$, and [deg] for $\delta$.

\begin{tabular}{c|cccccc}
\hline ID & Type & $\begin{array}{c}\log F_{X, \mathrm{obs}} \\
{\left[\mathrm{erg} \mathrm{s}^{-1} \mathrm{~cm}^{-2}\right]}\end{array}$ & $\begin{array}{c}\log L_{X, \mathrm{obs}} \\
{\left[\mathrm{erg} \mathrm{s}^{-1}\right]}\end{array}$ & $\begin{array}{c}\log M_{\mathrm{BH}} \\
{\left[\mathrm{M}_{\odot}\right]}\end{array}$ & $\begin{array}{c}d_{L} \\
{[\mathrm{Mpc}]}\end{array}$ & $\begin{array}{c}\delta \\
{[\mathrm{deg}]}\end{array}$ \\
\hline 4565 & $\mathrm{~S} 1.9$ & -10.73 & 41.32 & 7.43 & 9.7 & 26.0 \\
3516 & $\mathrm{~S} 1.2$ & -10.84 & 42.42 & 8.07 & 38.9 & 72.6 \\
4258 & $\mathrm{~S} 1.9$ & -10.84 & 40.90 & 7.62 & 6.8 & 47.3 \\
3227 & $\mathrm{~S} 1.5$ & -11.06 & 41.64 & 7.43 & 20.6 & 19.9 \\
4138 & $\mathrm{~S} 1.9$ & -11.26 & 41.28 & 7.17 & 17.0 & 43.7 \\
3169 & $\mathrm{~L} 2$ & -11.32 & 41.35 & 8.16 & 19.7 & 3.5 \\
4579 & $\mathrm{~S} 1.9 / \mathrm{L}$ & -11.36 & 41.17 & 7.86 & 16.8 & 11.8 \\
3998 & $\mathrm{~L} 1.2$ & -11.43 & 41.32 & 9.23 & 21.6 & 55.5 \\
3718 & $\mathrm{~L} 1.9$ & -11.48 & 41.06 & 7.77 & 17.0 & 53.1 \\
4203 & $\mathrm{~L} 1.9$ & -11.68 & 40.37 & 7.89 & 9.7 & 33.2 \\
4486 & $\mathrm{~L} 2$ & -11.71 & 40.82 & 9.42 & 16.8 & 12.4 \\
3031 & $\mathrm{~S} 1.5$ & -11.71 & 39.48 & 7.82 & 3.6 & 69.1 \\
\hline 5866 & $\mathrm{~T} 2$ & -14.16 & 38.29 & 7.92 & 15.3 & 55.8 \\
\hline
\end{tabular}

Compton- $Y$ parameter, which is consistent with the observed anti-correlation between the $\mathrm{X}$-ray spectral index and the Eddington ratio [123, 124].

Recently, Ref. [125] estimated the cutoff energy in Xray spectrum in NGC 3998 to be around $100 \mathrm{keV}$ using the NuSTAR and XMM-Newton data. However, they just measured a slight softening of the spectrum, which can be reconciled by our RIAF model. The Compton scattering makes a few bumps in the broad-band spectrum, which causes a softening in the X-ray band for NGC 3998 as seen in Figure 1] Here, we do not compare our model to observations in detail, because they are beyond the scope of this paper.

In order to obtain the electron temperature more concretely, we need to detect a clear cutoff feature above 100 $\mathrm{keV}$. We plot the photon spectra due to thermal electrons above $10 \mathrm{keV}$ with the sensitivity curve of the proposed future satellite, e-ASTROGAM [126] in Figure 3] The $\mathrm{MeV}$ gamma rays will be easily detected for NGC 3516 and NGC 4258, although it is not expected for NGC 3031. The other proposed MeV gamma-ray satellites, AMEGO [127] and GRAMS [128], have similar or better sensitivity in this range. The MeV observations of nearby LLAGNs will provide not only the electron temperature in RIAFs for the first time, but also the crucial test for the LLAGN contribution to the $\mathrm{MeV}$ gamma-ray background (see the accompanying paper).

\section{NON-THERMAL PROTONS IN RIAFS}

High-energy protons may be accelerated and injected into RIAFs by magnetic reconnections [102, 115, 129 132., stochastic acceleration via MHD turbulence 99,

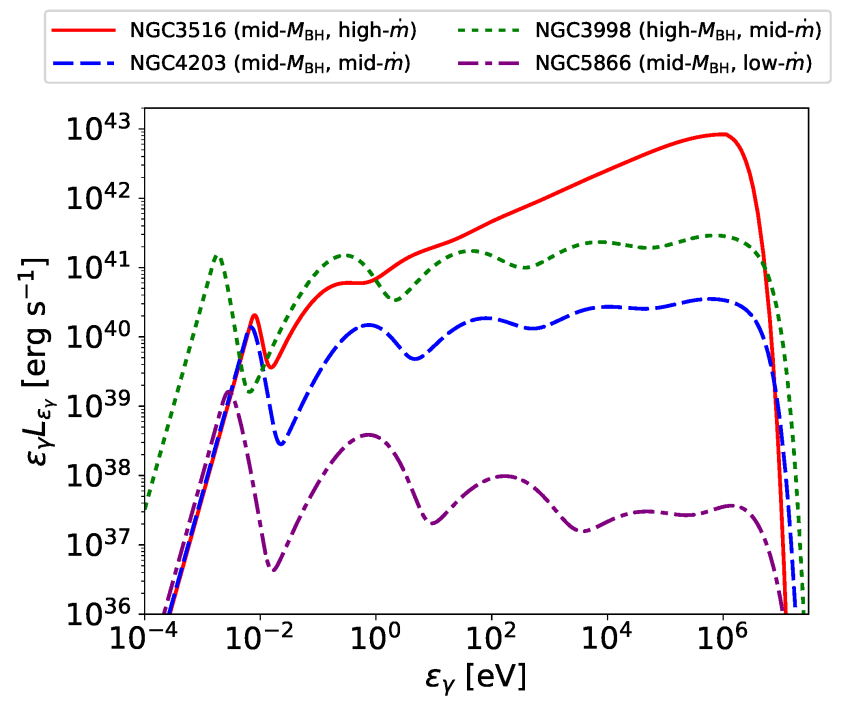

FIG. 1. Soft photon spectra for NGC 3516 (red-solid line), NGC 4203 (blue-dashed line), NGC 3998 (green-dotted line), and NGC 5866 (purple-dot-dashed).

133 135], or electric potential gaps in the black hole magnetosphere [136, 137]. We examine three cases of nonthermal proton spectra. One is the stochastic acceleration model (model A), in which we solve the diffusion equation in momentum space. The others are the powerlaw injection models (models B and C) in which we consider an injection term with a single power-law with an exponential cutoff. Such a power-law model mimics a generic acceleration process.

\section{A. Plasma condition}

For stochastic acceleration via turbulence to work, the relaxation time in the RIAF needs to be longer than the dissipation time, i.e., the plasma is collisionless. The relaxation time due to Coulomb collisions is estimated to be (e.g. Refs. [138, 139])

$$
\begin{aligned}
t_{\mathrm{rlx}} \approx & \frac{4 \sqrt{\pi}}{\ln \Lambda n_{p} \sigma_{T} c}\left(\frac{m_{p}}{m_{e}}\right)^{2}\left(\frac{C_{s}}{c}\right)^{3} \\
& \simeq 1.8 \times 10^{8} \alpha_{-1} M_{8} \dot{m}_{-2}^{-1} \mathrm{~s}
\end{aligned}
$$

where $\ln \Lambda \sim 20$ is the Coulomb logarithm. Interestingly, the relaxation time is independent of the normalized radius, $\mathcal{R}$. The dissipation time in the accretion flow is represented as $t_{\text {diss }} \sim \alpha^{-1} R / V_{K}$ [106, 140]. In the RIAF, this timescale is of the order of the infall time:

$$
t_{\text {fall }} \approx \frac{R}{V_{R}} \simeq 8.8 \times 10^{5} \mathcal{R}_{1}^{3 / 2} \alpha_{-1}^{-1} M_{8} \mathrm{~s} .
$$

Equating these two timescales, we obtain the critical radius within which the flow becomes collisionless (see also 
TABLE II. Physical quantities of the RIAF in the nearby LLAGNs. The values of $L_{p}$ and $P_{\mathrm{CR}} / P_{g}$ are for models A/B/C. Units are $[\mathrm{cm}]$ for $R,\left[\mathrm{~cm}^{-3}\right]$ for $n_{p},[\mathrm{G}]$ for $B,[\mathrm{MeV}]$ for $\varepsilon_{\gamma \gamma}$, and $\left[\mathrm{erg} \mathrm{s}^{-1}\right]$ for $L_{p}$.

\begin{tabular}{|c||c|c|c|c|c|c|c|c|c|}
\hline $\begin{array}{c}\text { ID } \\
\text { NGC }\end{array}$ & $\log \dot{m}$ & $\begin{array}{c}\log R \\
{[\mathrm{~cm}]}\end{array}$ & $\begin{array}{c}\log n_{p} \\
{\left[\mathrm{~cm}^{-3}\right]}\end{array}$ & $\begin{array}{c}\log B \\
{[\mathrm{G}]}\end{array}$ & $\log \tau_{T}$ & $\theta_{e}$ & $\begin{array}{c}\log \varepsilon_{\gamma \gamma} \\
{[\mathrm{MeV}]}\end{array}$ & $\begin{array}{c}\log L_{p} \\
{\left[\mathrm{erg} \mathrm{s}^{-1}\right]}\end{array}$ & $\begin{array}{c}P_{\mathrm{CR}} / P_{g} \\
{[\%]}\end{array}$ \\
\hline 4565 & -1.78 & 13.90 & 9.45 & 2.81 & -0.83 & 1.09 & 2.78 & $41.23 / 41.05 / 41.74$ & $10 / 6 / 37$ \\
3516 & -1.55 & 14.54 & 9.04 & 2.61 & -0.60 & 0.93 & 2.22 & $42.10 / 41.92 / 42.61$ & $8 / 4 / 29$ \\
4258 & -2.08 & 14.09 & 8.96 & 2.57 & -1.13 & 1.39 & 3.50 & $41.11 / 40.94 / 41.63$ & $12 / 8 / 44$ \\
3227 & -1.62 & 13.90 & 9.61 & 2.89 & -0.67 & 0.96 & 2.39 & $41.39 / 41.21 / 41.90$ & $9 / 5 / 32$ \\
4138 & -1.67 & 13.64 & 9.82 & 3.00 & -0.72 & 0.99 & 2.51 & $41.08 / 40.90 / 41.59$ & $9 / 6 / 34$ \\
3169 & -2.13 & 14.63 & 8.37 & 2.27 & -1.18 & 1.47 & 3.63 & $41.61 / 41.43 / 42.13$ & $12 / 8 / 44$ \\
4579 & -2.07 & 14.33 & 8.73 & 2.45 & -1.12 & 1.39 & 3.48 & $41.37 / 41.19 / 41.89$ & $12 / 8 / 43$ \\
3998 & -2.68 & 15.70 & 6.75 & 1.46 & -1.73 & 2.25 & 4.52 & $42.13 / 41.95 / 42.65$ & $14 / 10 / 50$ \\
3718 & -2.08 & 14.24 & 8.81 & 2.49 & -1.13 & 1.39 & 3.50 & $41.27 / 41.09 / 41.79$ & $12 / 8 / 43$ \\
4203 & -2.48 & 14.36 & 8.29 & 2.23 & -1.53 & 1.84 & 4.12 & $40.98 / 40.81 / 41.51$ & $14 / 9 / 49$ \\
4486 & -3.02 & 15.89 & 6.22 & 1.20 & -2.07 & 2.74 & 5.56 & $41.97 / 41.80 / 42.50$ & $15 / 10 / 52$ \\
3031 & -2.89 & 14.29 & 7.95 & 2.06 & -1.94 & 2.30 & 5.14 & $40.50 / 40.33 / 41.03$ & $15 / 10 / 52$ \\
\hline 5866 & -3.54 & 14.39 & 7.20 & 1.69 & -2.59 & 2.85 & 5.89 & $39.96 / 39.82 / 40.58$ & $16 / 12 / 66$ \\
\hline
\end{tabular}

TABLE III. Parameters in our models.

\begin{tabular}{ccccc}
\multicolumn{4}{c}{ Common parameters } \\
\hline$\alpha$ & $\beta$ & $\mathcal{R}$ & $\kappa_{\text {bol } / X}$ & $\epsilon_{\mathrm{rad}, \mathrm{sd}}$ \\
\hline 0.1 & 3.2 & 10 & 15 & 0.1
\end{tabular}

Model-dependent parameters and quantities

\begin{tabular}{cccccc}
\hline Parameters & $\epsilon_{p}$ & $\zeta$ & $q$ & $s_{\text {inj }}$ & $\eta_{\text {acc }}$ \\
\hline Model A & $3.0 \times 10^{-3}$ & $7.5 \times 10^{-3}$ & 1.666 & - & - \\
Model B & $2.0 \times 10^{-3}$ & - & - & 1.0 & $1.0 \times 10^{6}$ \\
Model C & 0.010 & - & - & 2.0 & $2.0 \times 10^{5}$ \\
\hline
\end{tabular}

Ref. [105]):

$$
\mathcal{R}_{\mathrm{crit}} \simeq 35 \alpha_{-1}^{4 / 3} \dot{m}_{-2}^{-2 / 3}
$$

As long as $\dot{m} \lesssim \dot{m}_{\text {crit }}$ with a fixed value of $\alpha \gtrsim 0.1$, the RIAF consists of collisionless plasma at $R \lesssim 10 R_{S}$. Hence, one may naturally expect non-thermal particle production there. On the other hand, another accretion regime with a higher luminosity, such as the standard disk [79] and the slim disk 141], are made up of collisional plasma because the density and temperature there are orders of magnitude higher and lower than that in the RIAF, respectively. Therefore, particle acceleration is not guaranteed due to the thermalization via Coulomb collisions.

\section{B. Stochastic acceleration model (A)}

In the stochastic acceleration model, protons are accelerated through scatterings with the MHD turbulence. The proton spectrum is obtained by solving the diffusion equation in momentum space (e.g., Refs. [142, 143]):

$$
\frac{\partial \mathcal{F}_{p}}{\partial t}=\frac{1}{\varepsilon_{p}^{2}} \frac{\partial}{\partial \varepsilon_{p}}\left(\varepsilon_{p}^{2} D_{\varepsilon_{p}} \frac{\partial \mathcal{F}_{p}}{\partial \varepsilon_{p}}+\frac{\varepsilon_{p}^{3}}{t_{\text {cool }}} \mathcal{F}_{p}\right)-\frac{\mathcal{F}_{p}}{t_{\mathrm{esc}}}+\dot{\mathcal{F}}_{p, \text { inj }}
$$

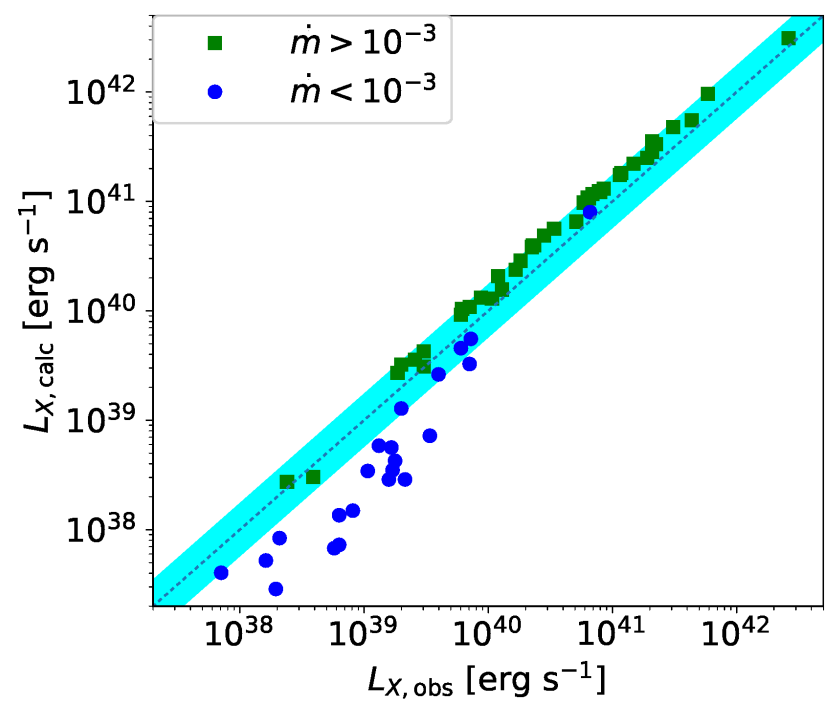

FIG. 2. Relationship between the observed X-ray luminosity, $L_{X \text {,obs }}$, and the X-ray luminosity obtained by the model

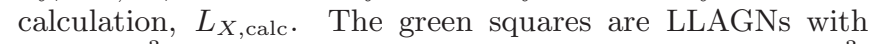
$\dot{m}>10^{-3}$, while the blue circles are those with $\dot{m}<10^{-3}$. The dotted line represents $L_{X, \mathrm{obs}}=L_{X \text {, calc }}$, and cyan band indicates $L_{X \text {,obs }} / 1.7<L_{X \text {, calc }}<1.7 L_{X \text {,obs }}$, in which all the green squares are located.

where $\mathcal{F}_{p}$ is the momentum distribution function $\left(d N / d \varepsilon_{p}=4 \pi p^{2} \mathcal{F}_{p} / c\right), D_{\varepsilon_{p}}$ is the diffusion coefficient, $t_{\text {cool }}$ is the cooling time, $t_{\text {esc }}$ is the escape time, and $\dot{\mathcal{F}}_{p \text {,inj }}$ is the injection term to the stochastic acceleration. Considering resonant scatterings with Alfven waves, the diffusion coefficient is represented as 144 146]

$$
D_{\varepsilon_{p}} \approx \frac{\zeta c}{H}\left(\frac{V_{A}}{c}\right)^{2}\left(\frac{r_{L}}{H}\right)^{q-2} \varepsilon_{p}^{2},
$$



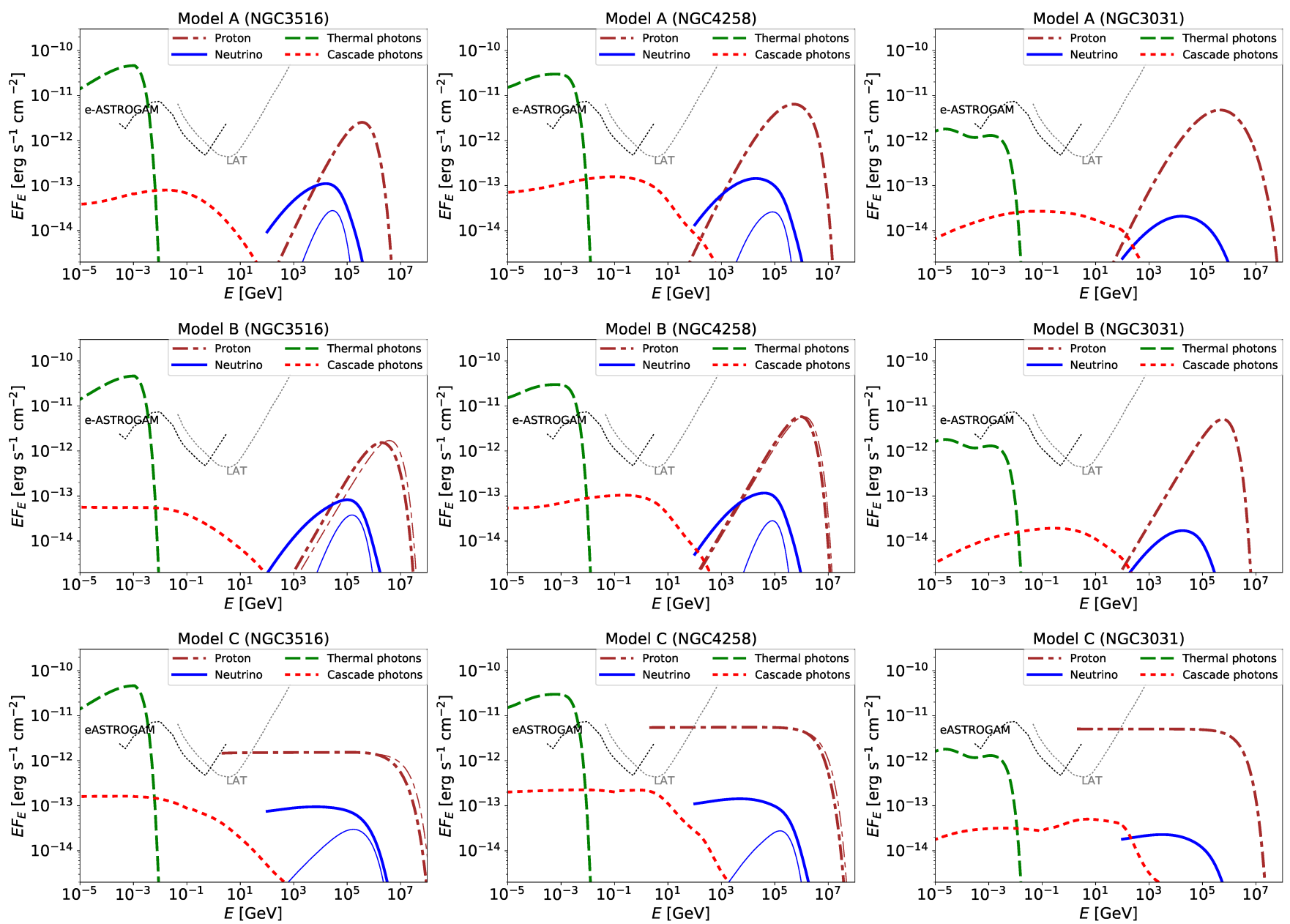

FIG. 3. Spectral energy distributions of gamma-ray (dashed by thermal electrons; dotted by hadronic cascade), neutrino (solid), and proton (dot-dashed) fluxes for Model A (stochastic acceleration; left panel), Model B (power-law injection with $s_{\text {inj }}=1.0$; middle), and Model $\mathrm{C}$ (power-law injection with $s_{\mathrm{inj}}=2.0$; right), respectively. The upper, middle, and lower panels are for NGC 3516, NGC 4258, and NGC 3031, respectively. The thin-dot-dashed lines in the middle and right columns depict the injection spectrum of the protons. The thin-solid lines are the $p \gamma$ neutrino flux. For NGC 3031, the $p \gamma$ neutrino flux is below the lower end of the figure.

where $r_{L}=\varepsilon_{p} /(e B)$ is the Larmor radius, $\zeta \approx$ $8 \pi \int P_{k} d k / B^{2}$ is the turbulent strength parameter, and $q$ is the power-law index of the turbulence power spectrum. The acceleration time is given by $t_{\mathrm{acc}} \approx \varepsilon_{p}^{2} / D_{\varepsilon_{p}}$. We use a delta-function injection: $\dot{\mathcal{F}}_{p \text {,inj }}=\dot{\mathcal{F}}_{0} \delta\left(\varepsilon_{p}-\varepsilon_{\text {inj }}\right)$, where $\dot{\mathcal{F}}_{0}$ is normalization factor. We normalize the luminosity of the non-thermal protons so that the proton luminosity is a constant fraction of the accretion luminosity:

$$
\int L_{\varepsilon_{p}} d \varepsilon_{p}=\epsilon_{p} \dot{m} L_{\mathrm{Edd}}
$$

where $L_{\varepsilon_{p}}=\varepsilon_{p} t_{\text {loss }}^{-1} d N / d \varepsilon_{p}$ is the differential proton luminosity $\left(t_{\text {loss }}^{-1}=t_{\text {cool }}^{-1}+t_{\text {esc }}^{-1}\right.$ is the total loss rate) and $\epsilon_{p}$ is the non-thermal proton production efficiency. We use the Chang \& Cooper method to solve the equation [147, 148], and calculate the time evolution until steady state is achieved. Note that the normalization is different from that used in Ref. [87], where we normalized the injection such that $\dot{\mathcal{F}}_{0}=f_{\text {inj }} L_{X, \text { obs }} /\left(4 \pi^{2} \varepsilon_{\text {inj }}^{3} R^{3}\right)$. Here, $f_{\text {inj }}$ is the efficiency of the injection to the stochastic acceleration, and $f_{\text {inj }}$ needs to be much smaller than $\epsilon_{p}$.

\section{Power-law injection models (B and C)}

For models B and C, we consider a generic acceleration mechanism, and the steady-state proton spectrum, $N_{\varepsilon_{p}}=d N / d \varepsilon_{p}$, is obtained by solving the transport equation:

$$
\frac{d}{d \varepsilon_{p}}\left(-\frac{\varepsilon_{p}}{t_{\text {cool }}} N_{\varepsilon_{p}}\right)=\dot{N}_{\varepsilon_{p}, \text { inj }}-\frac{N_{\varepsilon_{p}}}{t_{\text {esc }}}
$$


where $\dot{N}_{\varepsilon_{p}, \text { inj }}$ is the injection function. We consider a power-law injection with an exponential cutoff:

$$
\dot{N}_{\varepsilon_{p}, \text { inj }}=\dot{N}_{0}\left(\frac{\varepsilon_{p}}{\varepsilon_{p, \text { cut }}}\right)^{-s_{\text {inj }}} \exp \left(-\frac{\varepsilon_{p}}{\varepsilon_{p, \text { cut }}}\right)
$$

where $\dot{N}_{0}$ is the normalization factor, $s_{\text {inj }}$ is the injection spectral index, and $\varepsilon_{p \text {,cut }}$ is the cutoff energy. We normalize the injection by

$$
\int \varepsilon_{p} \dot{N}_{\varepsilon_{p}, \text { inj }} d \varepsilon_{p}=\epsilon_{p} \dot{m} L_{\mathrm{Edd}} .
$$

We can get an analytic solution of the transport equation (cf., Ref. [149]):

$$
\begin{gathered}
N_{\varepsilon_{p}}=\frac{t_{\mathrm{cool}}}{\varepsilon_{p}} \int_{\varepsilon_{p}}^{\infty} d \varepsilon_{p}^{\prime} \dot{N}_{p, \text { inj }}\left(\varepsilon_{p}^{\prime}\right) \exp \left(-\mathcal{G}\left(\varepsilon_{p}, \varepsilon_{p}^{\prime}\right)\right), \\
\mathcal{G}\left(\varepsilon_{1}, \varepsilon_{2}\right)=\int_{\varepsilon_{1}}^{\varepsilon_{2}} \frac{t_{\mathrm{cool}}}{t_{\mathrm{esc}}} \frac{d \varepsilon_{p}^{\prime}}{\varepsilon_{p}^{\prime}}
\end{gathered}
$$

This solution includes exponential term, so we need to carefully treat the numerical integration. In the rest of this paper, we show the results using Simpson's rule and 115 grid points per energy decade. We computed the numerical integration with the trapezoidal rule and/or with 50-200 grid points per decade, and confirmed that the error is reduced to less than $30 \%$ using Simpson's rule with 100 grid points per energy decade.

The maximum achievable energy of protons is determined by the balance between acceleration and loss. We phenomenologically write the acceleration time as

$$
t_{\mathrm{acc}} \approx \eta_{\mathrm{acc}} \frac{r_{L}}{c}
$$

where $\eta_{\text {acc }}$ is a parameter for the acceleration timescale. Since the infall is the most efficient loss process for majority of the LLAGNs, we estimate the cutoff energy by $t_{\text {acc }}=t_{\text {fall }}$. This treatment approximates the cutoff energy within an error of a factor of a few.

\section{Escape and cooling timescales}

High-energy protons escape from the RIAF via advection or diffusion. The advective escape time is equal to the infall time given by Equation (6). The diffusive escape time depends on the magnetic field configuration. According to MHD simulations, the magnetic fields in RIAFs are stretched to the azimuthal direction. The non-thermal protons' mean free path perpendicular to the magnetic field is much shorter than that along the field line (e.g., Refs. [99, 133] ). In the turbulence with a power spectrum of $P_{k} \propto k^{-q}$, the parallel mean free path and the perpendicular diffusion coefficient are estimated to be (e.g., Refs. 145, 146, 150, 151])

$$
\begin{aligned}
\lambda_{\|} & \approx \frac{r_{L}}{3 \zeta}\left(\frac{H}{r_{L}}\right)^{q-1}, \\
D_{\perp} & \approx \frac{D_{\|}}{1+\left(\lambda_{\|} / r_{L}\right)^{2}} .
\end{aligned}
$$

The Larmor radius in the RIAF is estimated to be

$$
r_{L} \simeq 1.3 \times 10^{10} \varepsilon_{p, 15} \mathcal{R}_{1}^{-5 / 4} \alpha_{-1}^{-1 / 2} M_{8}^{-1 / 2} \dot{m}_{-2}^{1 / 2} \beta_{0.5}^{-1 / 2} \mathrm{~cm},
$$

with our fiducial parameter set (see Table【II) and $\varepsilon_{p, 15}=$ $\varepsilon_{p} / \mathrm{PeV}$. Then, we obtain $\lambda_{\|} / r_{L} \simeq 2.3 \times 10^{4}$, leading to $D_{\perp} / D_{\|} \simeq 1.9 \times 10^{-9}$. Hence, we ignore the diffusive escape process in this paper, i.e., we use $t_{\text {esc }}=t_{\text {fall }}$. The value of $D_{\perp}$ could be larger due to possible cross-field diffusion. To understand the behavior of high-energy protons in configuration space, much more elaborate calculations would be required, which are beyond the scope of this paper (see Ref. [99] for related discussion).

As the proton cooling processes, we take into account $p p$ inelastic collisions, photomeson production, proton synchrotron processes, and the Bethe-Heitler process. The $p p$ cooling rate is

$$
t_{\mathrm{pp}}^{-1} \approx n_{p} \sigma_{p p} c \kappa_{p p}
$$

where $\sigma_{p p}$ and $\kappa_{p p}$ are the cross section and inelasticity for $p p$ interactions, respectively. $\sigma_{p p}$ was given in Ref. [152], and $\kappa_{p p}$ is set to be 0.5 . The photomeson production rate is

$$
t_{p \gamma}^{-1}=\frac{c}{2 \gamma_{p}^{2}} \int_{\bar{\varepsilon}_{\mathrm{th}}}^{\infty} d \bar{\varepsilon}_{\gamma} \sigma_{p \gamma} \kappa_{p \gamma} \bar{\varepsilon}_{\gamma} \int_{\bar{\varepsilon}_{\gamma} /\left(2 \gamma_{p}\right)}^{\infty} d \varepsilon_{\gamma} \varepsilon_{\gamma}^{-2} \frac{d n_{\gamma}}{d \varepsilon_{\gamma}},
$$

where $\gamma_{p}=\varepsilon_{p} /\left(m_{p} c^{2}\right), \bar{\varepsilon}_{p, \text { th }} \simeq 145 \mathrm{MeV}$ is the threshold energy for the photomeson production, $\bar{\varepsilon}_{\gamma}$ is the photon energy in the proton rest frame, and $\sigma_{p \gamma}$ and $\kappa_{p \gamma}$ are the cross section and inelasticity for photomeson production, respectively. We use fitting formulas based on GEANT4 for $\sigma_{p \gamma}$ and $\kappa_{p \gamma}$ (see Ref. [11]). The Bethe-Heitler cooling rate is also estimated by Equation (21) using $\sigma_{\mathrm{BH}}$ and $\kappa_{\mathrm{BH}}$ instead of $\sigma_{p \gamma}$ and $\kappa_{p \gamma}$, respectively. We use the fitting formulas given in Refs. 153 and 154 for $\sigma_{\mathrm{BH}}$ and $\kappa_{\mathrm{BH}}$, respectively. The synchrotron cooling rate is estimated to be

$$
t_{\mathrm{syn}}^{-1}=\frac{\gamma_{p} \sigma_{T} B^{2}}{6 \pi m_{p} c}\left(\frac{m_{e}}{m_{p}}\right)^{2} .
$$

The total cooling rate is given by the sum of all the cooling rates.

Figure 4 shows the loss and acceleration rates as a function of proton energy for NGC 3516, NGC 4258, and NGC3031, which have $\dot{m} \sim 0.9 \dot{m}_{\mathrm{cr}}, \dot{m} \sim 0.3 \dot{m}_{\mathrm{cr}}$, and $\dot{m} \sim 0.04 \dot{m}_{\text {cr }}$ respectively. For NGC $3516, t_{\text {fall }}$ and $t_{p p}$ are comparable in the entire energy range. The photomeson production is effective above $\varepsilon_{p} \gtrsim 30 \mathrm{PeV}$. The synchrotron and Bethe-Heitler losses are always subdominant in the range of our interest. On the other hand, for 
NGC 4258 and NGC 3031, the infall timescale is always dominant below the cutoff energy due to lower $\dot{m}$. Note that the critical energy at which $t_{\mathrm{acc}}=t_{\text {loss }}$ is very low for model A, compare to the other models. Such a lower critical energy is required to achieve a cutoff energy similar to the other models (see Figure 3) because the stochastic acceleration results in a hard spectrum with a gradual cutoff (cf. Refs. 91, 155]).

To understand the parameter dependences of each timescale, we write $t_{\mathrm{p} \gamma}^{-1} \sim n_{\varepsilon_{\gamma}} \kappa_{p \gamma} \sigma_{p \gamma} c$, where $n_{\varepsilon_{\gamma}} \approx$ $L_{\varepsilon_{\gamma}} /\left(2 \pi R^{2} c \varepsilon_{\gamma}\right)$ is the differential photon number density and $L_{\varepsilon_{\gamma}}$ is the differential photon luminosity. Then, if we fix the parameters in Table III the parameter dependence of the loss rates are $t_{\text {fall }}^{-1} \propto M_{\mathrm{BH}}^{-1}, t_{p p}^{-1} \propto \dot{m} M_{\mathrm{BH}}^{-1}$, $t_{p \gamma}^{-1} \propto \dot{m}^{2} M_{\mathrm{BH}}^{-1}$, and $t_{\mathrm{syn}}^{-1} \propto \dot{m} M_{\mathrm{BH}}^{-1}$. Interestingly, all the loss rates are proportional to $M_{\mathrm{BH}}^{-1}$, while they have a different $\dot{m}$ dependence. For the case with $\dot{m} \sim \dot{m}_{\text {crit }}$ as in NGC 3516, $t_{p \gamma}^{-1} \lesssim t_{p p}^{-1}$ and $t_{p p}^{-1} \sim t_{\text {fall }}$ below the cutoff energy. Since a lower value of $\dot{m}$ makes $t_{\text {fall }}$ shorter and $t_{p \gamma}$ longer relative to $t_{p p}$, we can approximately use $t_{\text {fall }}$ as the energy loss timescale, and $p p$ collisions are the main channel of neutrino production for $\dot{m} \lesssim \dot{m}_{\text {crit }}$. We describe analytic estimates with this approximation in Section IV]

Figure 3 shows the resulting proton spectrum, $E_{p} F_{E_{p}}=\varepsilon_{p} L_{\varepsilon_{p}} /\left(4 \pi d_{L}^{2}\right)$, and the injection proton spectrum, $E_{p} F_{E_{p}, \text { inj }}=\varepsilon_{p}^{2} \dot{N}_{\varepsilon_{p}, \text { inj }} /\left(4 \pi d_{L}^{2}\right)$, where $E_{p}$ is the energy in the observer's frame. Since we focus on the very nearby objects, we ignore the effect of redshift, i.e., $E_{p} \approx \varepsilon_{p}$. The parameter sets are tabulated in Tables \ and III] We choose these parameter sets so that our model can reproduce the diffuse $\mathrm{MeV}$ gamma-ray and $\mathrm{TeV}-\mathrm{PeV}$ neutrino intensities (see the accompanying paper). We also tabulate the total proton luminosity, $L_{p}=\int L_{\varepsilon_{p}} d \varepsilon_{p}$, and pressure ratio of the non-thermal to thermal components, $P_{\mathrm{CR}} / P_{g}=\int \varepsilon_{p} N_{\varepsilon_{p}} d \varepsilon_{p} /\left(6 \pi R^{2} H m_{p} n_{p} C_{s}^{2}\right)$. To achieve the observed diffuse neutrino intensity, we need $P_{\mathrm{CR}} / P_{g} \sim 0.1$ for models $\mathrm{A}$ and $\mathrm{B}$, while $P_{\mathrm{CR}} / P_{g} \sim 0.5$ for model $\mathrm{C}$.

In model $\mathrm{A}$, the stochastic acceleration model leads to a hard spectrum below the critical energy, which is $E_{p} F_{E_{p}} \propto \varepsilon_{p}^{3-q}$. Above the critical energy, the spectrum gradually becomes softer. For NGC 3516, the photomeson production is efficient above $\varepsilon_{p} \simeq 10^{6} \mathrm{GeV}$, which makes a sharp cutoff. For NGC 4258 and NGC 3031, the cooling processes are inefficient. This leads to a more gradual cutoff, resulting in a higher peak energy than that for NGC 3516. In models B and C, the resulting spectra are very similar to the injection spectra, because the infall is the dominant loss process. In this case, the proton number spectrum in the RIAF is written as $N_{\varepsilon_{p}} \approx \dot{N}_{\varepsilon_{p}, \text { inj }} t_{\text {fall }}$, leading to $L_{\varepsilon_{p}} \approx \varepsilon_{p} \dot{N}_{\varepsilon_{p}, \text { inj }}$. For NGC 3516 , we can see a slight difference between the two spectra due to the $p p$ cooling. Note that we cannot observe this flux of protons on Earth because of the energy loss processes and deflection by interstellar and intergalactic magnetic fields.

\section{HIGH-ENERGY NEUTRINOS}

\section{A. Meson cooling}

We numerically calculate the neutrino production through both photomeson and hadronuclear interactions. The neutrinos are produced by decay of pions and muons. In general the high-energy neutrinos can be suppressed by meson and muon cooling, when their lifetimes are longer than the cooling time. Here, we estimate the hadronic cooling time for pions and synchrotron cooling for pions and muons. The hadronic cooling rate for pions is estimated to be $t_{\pi p}^{-1} \sim n_{p} \sigma_{\pi p} \kappa_{\pi p} c$, where $\sigma_{\pi p} \sim 50$ $\mathrm{mb}$ and $\kappa_{\pi p} \sim 0.8$ are the pion-proton interaction cross section and inelasticity, respectively. The critical energy above which the pion hadronic cooling is efficient is $\varepsilon_{\nu, \pi \mathrm{p}} \approx m_{\pi} c^{2} /\left(n_{p} \sigma_{\pi p} \kappa_{\pi p} c \tau_{\pi 0}\right) \sim 2 \times 10^{21} \mathcal{R}_{1}^{3 / 2} \alpha_{-1} M_{8} \dot{m}_{-2}^{-1}$ $\mathrm{eV}$, where $m_{\pi}$ and $\tau_{\pi 0}$ are the mass and decay time of pions, respectively. Thus, we can safely ignore the pion hadronic cooling.

The synchrotron cooling time for a particle $i$ is written as $t_{i, \mathrm{syn}} \approx 6 \pi m_{i}^{5} c^{5} /\left(m_{e}^{2} \sigma_{T} c \varepsilon_{i}^{2} B^{2}\right)$, where $m_{i}$ and $\varepsilon_{i}$ are the mass and energy of the particle. Equating the lifetime and synchrotron cooling time, we can estimate the critical energies above which the synchrotron cooling is effective to be $\varepsilon_{\nu, \pi \mathrm{syn}}=\sqrt{3 \pi m_{\pi}^{5} c^{5} /\left(8 m_{e}^{2} \sigma_{T} B^{2} \tau_{\pi}\right)} \simeq$ $1.0 \times 10^{17} \mathcal{R}_{1}^{5 / 4} \alpha_{-1}^{1 / 2} \beta_{0.3}^{1 / 2} M_{8}^{1 / 2} \dot{m}_{-2}^{-1 / 2} \mathrm{eV}$ for pions and $\varepsilon_{\nu, \mu \text { syn }}=\sqrt{2 \pi m_{\mu}^{5} c^{5} /\left(3 m_{e}^{2} \sigma_{T} B^{2} \tau_{\mu}\right)} \simeq$ $7.5 \times 10^{15} \mathcal{R}_{1}^{5 / 4} \alpha_{-1}^{1 / 2} \beta_{0.5}^{1 / 2} M_{8}^{1 / 2} \dot{m}_{-2}^{-1 / 2} \mathrm{eV}$ for muons. Here, $m_{\mu}$ and $\tau_{\mu 0}$ are the mass and decay time of muons, respectively. Since we are interested in $\mathrm{TeV}-\mathrm{PeV}$ neutrinos, we will ignore the cooling effect by mesons and muons.

\section{B. Neutrino spectrum}

To calculate high-energy neutrino spectra from $p p$ interactions, we use the method given by Ref. [156], where the $p p$-neutrino spectrum, $L_{p p, \varepsilon_{\nu}}=\varepsilon_{\nu} t_{\mathrm{pp}}^{-1} d N / d \varepsilon_{\nu}$, is given by

$$
\frac{L_{p p, \varepsilon_{\nu}}}{\varepsilon_{\nu}} \approx c n_{p} \int_{\varepsilon_{\nu}}^{\infty} \sigma_{p p}\left(\varepsilon_{p}\right) N_{\varepsilon_{p}} \mathcal{H}_{\nu}\left(\frac{\varepsilon_{\nu}}{\varepsilon_{p}}, \varepsilon_{p}\right) \frac{d \varepsilon_{p}}{\varepsilon_{p}},
$$

where $\mathcal{H}_{\nu}\left(\varepsilon_{\nu} / \varepsilon_{p}, \varepsilon_{p}\right)$ is the spectral shape of the neutrinos from mono-energetic protons of $\varepsilon_{p}$ (see Ref. [156] for details). This method is valid only for $\varepsilon_{\nu}>100$ $\mathrm{GeV}$. Since our scope is to discuss the detection prospects by IceCube-like detectors, we focus on neutrinos above $100 \mathrm{GeV}$. For $p \gamma$ neutrinos, we approximately calculate the spectrum using the semi-analytic formalism of Refs. [17, 29], including the physical processes described in the previous section. Ignoring the effects of the meson cooling, the $p \gamma$-neutrino spectrum is given by

$$
\varepsilon_{\nu} L_{p \gamma, \varepsilon_{\nu}} \approx \frac{3}{8} f_{p \gamma} \varepsilon_{p} L_{\varepsilon_{p}},
$$



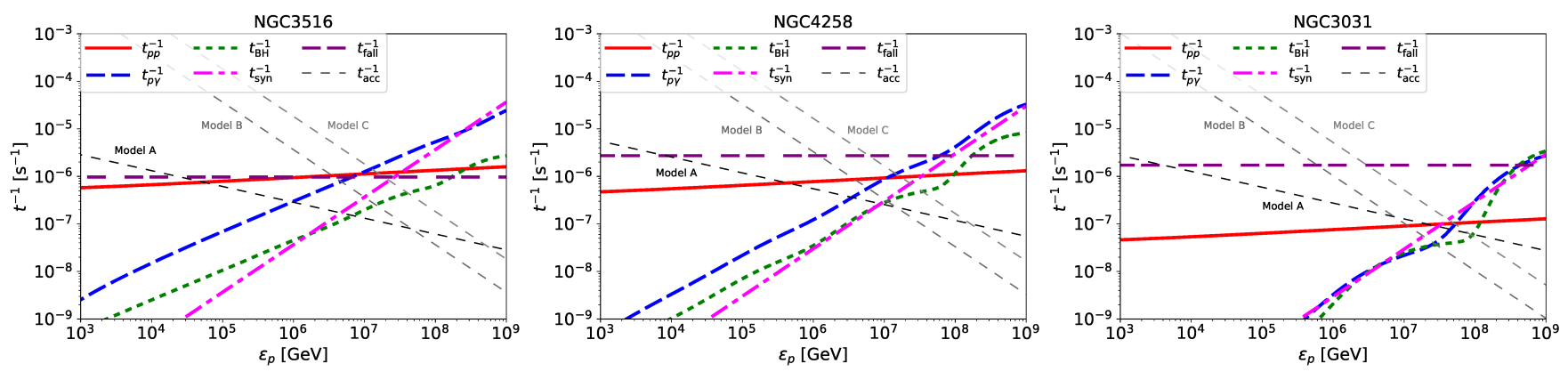

FIG. 4. The cooling, escape, and acceleration rates for NGC 3516 (left), NGC 4258 (middle), and NGC 3031 (right).

where $\varepsilon_{\nu} \approx 0.05 \varepsilon_{p}$ and $f_{p \gamma} \approx t_{p \gamma}^{-1} / t_{\text {loss }}^{-1}$. The neutrino flavor ratio at the sources is $\left(\nu_{e}, \nu_{\mu}, \nu_{\tau}\right)=(1,2,0)$ owing to the inefficient muon and pion cooling. The neutrinos change their flavors to $\left(\nu_{e}, \nu_{\mu}, \nu_{\tau}\right)=(1,1,1)$ during the propagation to the Earth through neutrino oscillation, and thus, the muon neutrino flux is a factor of 3 lower than the total neutrino flux.

Figure 3 shows the resulting muon neutrino fluxes,

$$
E_{\nu_{\mu}} F_{E_{\nu \mu}} \approx \frac{\varepsilon_{\nu} L_{\varepsilon_{\nu}}}{12 \pi d_{L}^{2}}
$$

where $L_{\varepsilon_{\nu}}=L_{p p, \varepsilon_{\nu}}+L_{p \gamma, \varepsilon_{\nu}}$. Since the $p p$ neutrino decay spectrum is softer than the parent proton spectrum for models A and B, these two models give similar neutrino spectral shapes. The neutrinos produced by $p p$ interaction are dominant for the low energy range, but the photomeson production gives a comparable contribution around the cutoff energy for the cases with $\dot{m} \gtrsim 0.01$ (NGC 3516 and NGC 4258). For NGC 3031, $\dot{m}$ is too low to effectively create neutrinos via photomeson production.

\section{Analytic estimate}

We can approximately derive analytic estimates of the neutrino flux from LLAGNs for the power-law injection cases. When infall is the dominant loss process, we can write $N_{\varepsilon_{p}} \approx t_{\text {fall }} \dot{N}_{p \text {,inj }}$, as discussed in the previous section. Then, the proton luminosity is approximated to be

$$
\varepsilon_{p} L_{\varepsilon_{p}} \approx \varepsilon_{p}^{2} \dot{N}_{p, \text { inj }}
$$

and the normalization is determined by $\int L_{\varepsilon_{p}} d \varepsilon_{p}=$ $\epsilon_{p} \dot{m} L_{\mathrm{Edd}} \propto \epsilon_{p} \dot{m} M_{\mathrm{BH}}$. The neutrino production efficiency is given by

$$
f_{p p} \approx \min \left(1, \frac{t_{\text {fall }}}{t_{p p}}\right) \approx \frac{8 \dot{m}}{\alpha^{2}} \frac{\sigma_{p p} \kappa_{p p}}{\sigma_{T}} \simeq 0.36 \alpha_{-1}^{-2} \dot{m}_{-2},
$$

where we use $\sigma_{p p} \sim 60 \mathrm{mb}$ and $\kappa_{p p} \sim 0.5$ for the estimate, which corresponds to the values for $\varepsilon_{p} \sim 1-10 \mathrm{PeV} . f_{p p}$ becomes unity around the saturation accretion rate,

$$
\dot{m}_{\text {sat }} \sim 2.8 \times 10^{-2} \alpha_{-1} .
$$

With our reference parameters, this accretion rate is very close to the critical accretion rate, $\dot{m}_{\text {crit }}$. The all-flavor differential neutrino luminosity is approximated to be

$$
\varepsilon_{\nu} L_{\varepsilon_{\nu}} \approx \frac{1}{2} f_{p p} \varepsilon_{p} L_{\varepsilon_{p}} \propto \epsilon_{p} \dot{m}^{2} \alpha^{-2} M_{\mathrm{BH}} \propto L_{X} \epsilon_{p},
$$

where $\varepsilon_{\nu} \approx 0.04 \varepsilon_{p}$. Interestingly, the neutrino luminosity is proportional to $L_{X}$ and $\epsilon_{p}$, and independent of the other parameters. The differential muon neutrino energy flux is computed using Equations (12), (25), (26), (27), and (29). This method approximates the peak $p p$ neutrino flux within an error of factors of 2 and 1.3 for $s_{\text {inj }}=1.0$ and 2.0 , respectively.

\section{Detectability of neutrinos from nearby LLAGNs}

We evaluate the number of through-going muon track events following Refs. [49, 157]. We estimate the differential detection rate of through-going tracks:

$$
\frac{d \dot{\mathcal{N}_{\mu}}}{d E_{\mu}} \approx \frac{\mathcal{N}_{A} \mathcal{A}_{\mathrm{det}}}{\alpha_{\mu}+\beta_{\mu} E_{\mu}} \int_{E_{\mu}}^{\infty} d E_{\nu} \phi_{\nu_{\mu}} \sigma_{\mathrm{CC}} e^{-\tau_{\nu N}}
$$

where $E_{\nu}$ is the incoming neutrino energy, $E_{\mu}$ is the muon energy, $\mathcal{N}_{A}$ is the Avogadro number, $\mathcal{A}_{\text {det }}$ is the muon effective area, $\sigma_{\mathrm{CC}}$ is the charged-current cross section, $\tau_{\nu N}$ is the optical depth to neutrino-nucleon scatterings in the Earth, and the denominator in the right-hand side indicates the muon energy loss rate (see Ref. 49] and references therein). This method can reproduce the effective area reported by Ref. [158]. We evaluate the background including both the conventional and the prompt atmospheric muon neutrinos.

We plot $\mathcal{N}_{\mu}\left(>E_{\mu}\right)=\int_{E_{\mu}}^{\infty} d E_{\mu}^{\prime} \int d t d \dot{\mathcal{N}}_{\mu} / d E_{\mu}^{\prime}$ in Figure 5 for a ten-year operation with IceCube and IceCubeGen2 for NGC 3516, NGC 4258, and NGC 3031. IceCube cannot detect signals from individual objects due to lower effective area. IceCube-Gen2 can detect the signals from 
NGC 4258, while it is challenging to detect NGC 3516. Although NGC 3516 has a neutrino flux comparable to that of NGC 4258, the higher declination causes the lower $N_{\mu}\left(>E_{\mu}\right)$ due to the Earth attenuation, especially in Model B. The neutrino emission from NGC 3031 is too faint to be detected even with IceCube-Gen2.

Since the neutrino flux is roughly proportional to the X-ray flux, we place the LLAGNs listed in Ref. [121] in order of the X-ray flux, as shown in Table【 and estimate the number of track events above $E_{\mu}$ by stacking them. Figure 6 shows the resulting event number for a 10-year operation with IceCube-Gen2 and IceCube by stacking 10 LLAGNs and 30 LLAGNs. With IceCube-Gen2, we expect $3-7$ events above $30 \mathrm{TeV}$ where the background is negligible. Interestingly, the neutrinos from the ten brightest LLAGNs will be sufficient for the detection, because stacking more LLAGNs leads to an increase of the atmospheric background. With the current IceCube experiment, the effective area and angular resolution are $10^{2 / 3}$ times smaller and $3-5$ times larger than those of IceCube-Gen2, respectively. Then, the event number is about $4-5$ times lower and the background rate is $10-$ 30 times higher, making the detection of neutrinos more challenging, as seen in the figures.

\section{CASCADE GAMMA-RAY EMISSION}

Hadronuclear and photohadronic processes produce very-high-energy (VHE) gamma rays through neutral pion decay and high-energy electron/positron pairs through charged pion decay and the Bethe-Heitler process. The VHE gamma rays are absorbed by soft photons through the $\gamma \gamma \rightarrow e^{+} e^{-}$process in the RIAF, and produce additional high-energy electron/positron pairs. The high-energy $e^{+} e^{-}$pairs also emit gammarays through synchrotron, inverse Compton scattering, and bremsstrahlung, leading to electromagnetic cascades. We calculate the cascade emission by solving the kinetic equations of photons and electron/positron pairs (see Refs. [87, 159, 160]):

$$
\begin{array}{r}
\frac{\partial n_{\varepsilon_{e}}^{e}}{\partial t}+\frac{\partial}{\partial \varepsilon_{e}}\left[\left(P_{\mathrm{IC}}+P_{\mathrm{syn}}+P_{\mathrm{ff}}+P_{\mathrm{Cou}}\right) n_{\varepsilon_{e}}^{e}\right] \\
=\dot{n}_{\varepsilon_{e}}^{(\gamma \gamma)}-\frac{n_{\varepsilon_{e}}^{e}}{t_{\mathrm{esc}}}+\dot{n}_{\varepsilon_{e}}^{\mathrm{inj}}, \\
\frac{\partial n_{\varepsilon_{\gamma}}^{\gamma}}{\partial t}=-\frac{n_{\varepsilon_{\gamma}}^{\gamma}}{t_{\gamma \gamma}}-\frac{n_{\varepsilon_{\gamma}}^{\gamma}}{t_{\mathrm{esc}}}+\dot{n}_{\varepsilon_{\gamma}}^{(\mathrm{IC})}+\dot{n}_{\varepsilon_{\gamma}}^{(\mathrm{ff})}+\dot{n}_{\varepsilon_{\gamma}}^{(\mathrm{syn})}+\dot{n}_{\varepsilon_{\gamma}}^{\mathrm{inj}},
\end{array}
$$

where $n_{\varepsilon_{i}}^{i}$ is the differential number density $(i=e$ or $\gamma), \dot{n}_{\varepsilon_{i}}^{(x x)}$ is the particle source term from the process $x x(x x=\mathrm{IC}$ (inverse Compton scattering), $\gamma \gamma(\gamma \gamma$ pair production), syn (synchrotron), or ff (bremsstrahlung)), $\dot{N}_{\varepsilon_{i}}^{\text {inj }}$ is the injection term from the hadronic interaction, and $P_{y y}$ is the energy loss rate for the electrons from the process $y y$ ( $y y=$ IC (inverse Compton scattering), syn (synchrotron), ff (bremsstrahlung), or Cou (Coulomb collision)). We calculate the cascade spectra using spherical coordinates, while the other calculations are made in cylindrical coordinates. The effect of geometry has little influence on our results.

Here, we approximately treat the injection terms of photons and pairs from hadronic interactions. The injection terms for photons and pairs consist of the sum of the relevant processes: $\dot{n}_{\varepsilon_{\gamma}}^{\text {inj }}=\dot{n}_{\varepsilon_{\gamma}}^{(p \gamma)}+\dot{n}_{\varepsilon_{\gamma}}^{(p p)}$ and $\dot{n}_{\varepsilon_{e}}^{\text {inj }}=\dot{n}_{\varepsilon_{e}}^{(\mathrm{BH})}+\dot{n}_{\varepsilon_{e}}^{(p \gamma)}+\dot{n}_{\varepsilon_{e}}^{(p p)}$. We approximate the terms due to Bethe-Heitler and $p \gamma$ processes to be

$$
\begin{aligned}
& \varepsilon_{\gamma}^{2} \dot{n}_{\varepsilon_{\gamma}}^{(p \gamma)} \approx \frac{1}{2} t_{p \gamma}^{-1} \varepsilon_{p}^{2} n_{\varepsilon_{p}}, \\
& \varepsilon_{e}^{2} \dot{n}_{\varepsilon_{e}}^{(p \gamma)} \approx \varepsilon_{\nu}^{2} n_{\varepsilon_{\nu}}^{(p \gamma)} \approx \frac{1}{8} t_{\mathrm{p} \gamma}^{-1} \varepsilon_{p}^{2} n_{\varepsilon_{p}}, \\
& \varepsilon_{e}^{2} \dot{n}_{\varepsilon_{e}}^{(\mathrm{BH})} \approx t_{\mathrm{BH}}^{-1} \varepsilon_{p}^{2} n_{\varepsilon_{p}},
\end{aligned}
$$

where $\varepsilon_{\gamma} \approx 0.1 \varepsilon_{p}$ and $\varepsilon_{e} \approx 0.05 \varepsilon_{p}$ for photomeson production, and $\varepsilon_{e} \approx\left(m_{e} / m_{p}\right) \varepsilon_{p}$ for Bethe-Heitler process. For the injection terms from $p p$ interactions, see Ref. [160].

We plot proton-induced cascade gamma-ray spectra in Figure 3 A sufficiently developed cascade emission generates a flat spectrum below the critical energy at which $\gamma \gamma$ attenuation becomes ineffective. The optical depth to the electron-positron pair production is estimated to be

$$
\tau_{\gamma \gamma}\left(\varepsilon_{\gamma}\right) \approx R \int \mathcal{K}(x) \frac{d n_{\gamma}}{d \varepsilon_{\gamma}} d \varepsilon_{\gamma},
$$

where $\varepsilon_{\gamma}$ is the gamma-ray energy, $\mathcal{K}(x)=0.652 \sigma_{T}(x-$ $\left.x^{-2}\right) \ln (x) H(x-1), x=\varepsilon_{\gamma} \varepsilon_{\gamma} /\left(m_{e} c^{2}\right)$, and $H(x)$ is the Heaviside step function [161]. We tabulate the values of the critical energy, $\varepsilon_{\gamma \gamma}$, at which $\tau_{\gamma \gamma}=1$ in Table [I. We can see flat spectra below the critical energy. Note that the tabulated values are approximately calculated using a fitting formula, while the cascade calculations are performed with the exact cross section. We overplot the Fermi LAT sensitivity curve in the high galactic latitude region with a 10-year exposure obtained from Ref. [126]. The predicted fluxes are lower than the sensitivity curve for all the cases. The Cherenkov Telescope Array (CTA) has a better sensitivity above $30 \mathrm{GeV}$ than LAT, but the cascade gamma-ray flux is considerably suppressed in the VHE range due to the $\gamma \gamma$ attenuation. For a lower $\dot{m}$ object that has a higher value of $\varepsilon_{\gamma \gamma}$, such as NGC 5866 , the cascade flux is too low to be detected by CTA. Therefore, it would be challenging to detect the cascade gamma rays with current and near-future instruments, except for Sgr A*.

Sgr $\mathrm{A}^{*}$ has two distinct emission phases: the quiescent and flaring states (see Ref. [162] for review). The $\mathrm{X}$-ray emission from the quiescent state of $\mathrm{Sgr} \mathrm{A}^{*}$ is spatially extended to $\sim 1$ ", which corresponds to $10^{5} R_{S}$ for a black hole of $4 \times 10^{6} \mathrm{M}_{\odot}$ [163]. Hence, our model is not applicable to the quiescent state. On the other hand, the flaring state of Sgr A* shows $10-300$ times higher flux than the quiescent state with the time variability 

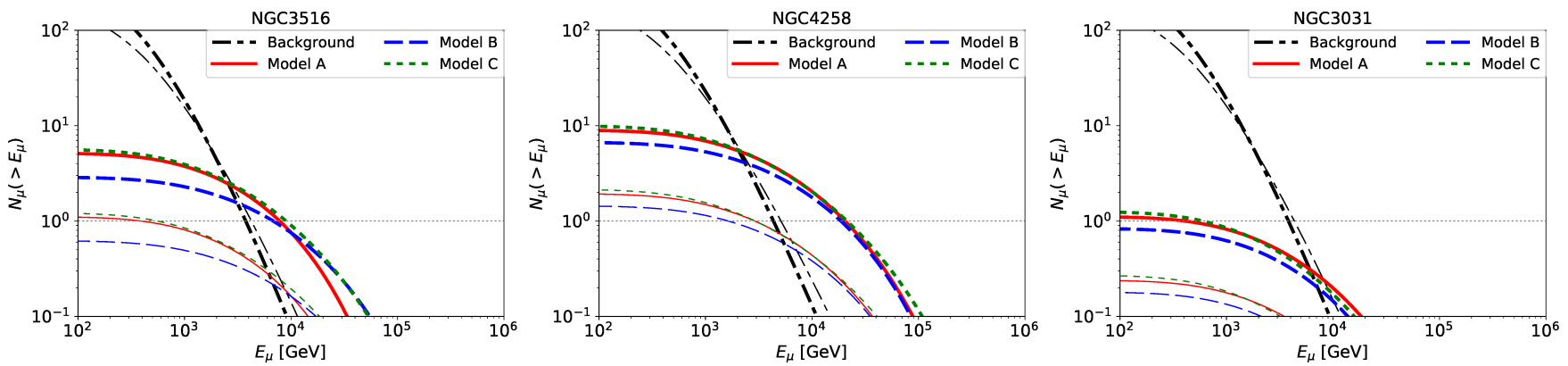

FIG. 5. The expected number of through-going track events from NGC 3516 (left panel), NGC 4258 (middle panel), and NGC 3031 (right panel) for models A (solid), B (dashed), and C (dotted) for a 10-year operation of IceCube-Gen2 (thick lines) and for IceCube (thin lines). The dot-dashed lines show the expected background.
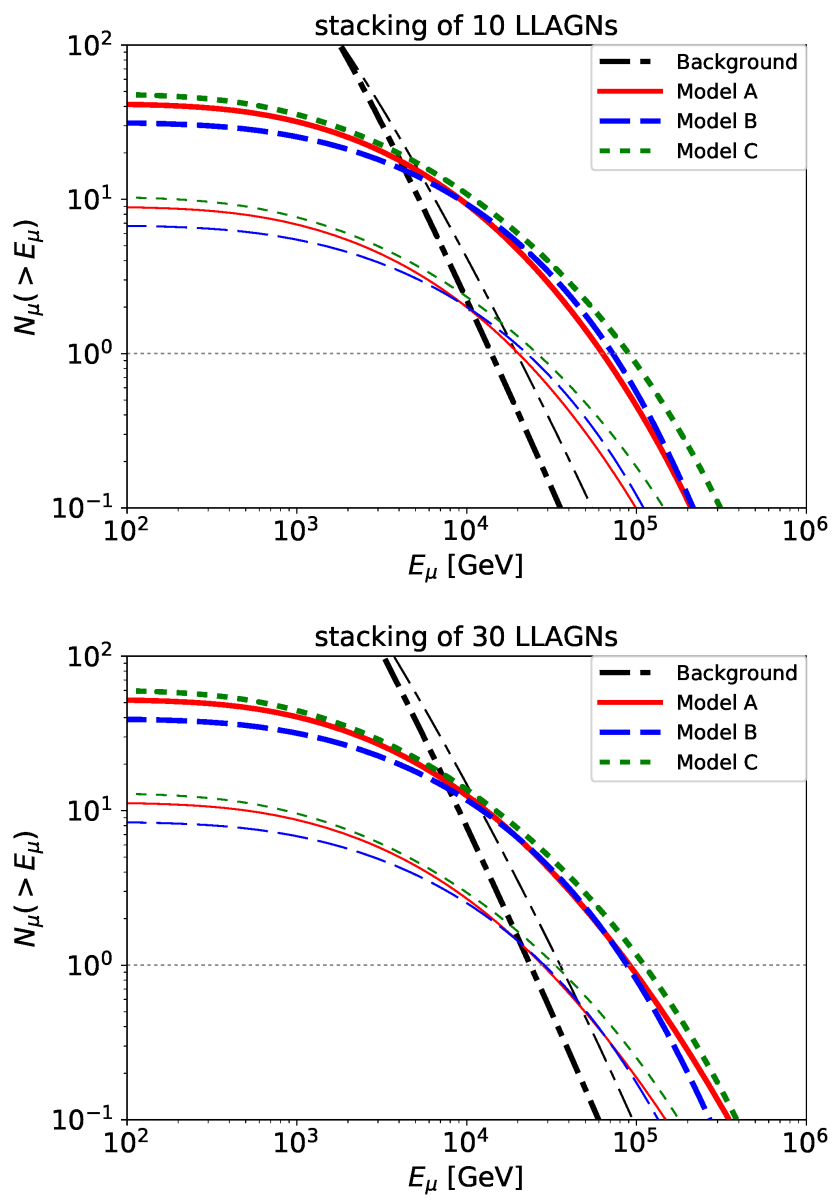

FIG. 6. Same as Figure 5 but stacking 10 (upper panel) and 30 LLAGNs (lower panel).

of $\sim 1 \mathrm{~h}$ [164]. This variability timescale implies that the emission region should be $\lesssim 10^{2} R_{S}$. However, the value of $\dot{m}$ for the brightest flare estimated by Equation (3) is less than $10^{-4}$. Since our model is not applicable to such a low-accretion-rate system (see Section ஹ), we avoid discussing it in detail. The detailed estimate should be made in the future (see Ref. [165] for related discussion).

\section{SUMMARY}

We have investigated high-energy multi-messenger emissions, including the MeV gamma-rays, high-energy gamma-rays, and neutrinos, from nearby individual LLAGNs, focusing on their multi-messenger detection prospects. We have refined the RIAF model of LLAGNs, referring to recent simulation results. Our one-zone model is roughly consistent with the observed X-ray features, such as an anti-correlation between the Eddington ratio and the spectral index. RIAFs with $\dot{m} \gtrsim 0.01$ emit strong $\mathrm{MeV}$ gamma rays through Comptonization, which will be detected by the future $\mathrm{MeV}$ satellites such as eASTROGAM, AMEGO, and GRAMS.

We have also calculated the neutrino and cascade gamma-ray spectra from accelerated protons. We considered three models for the proton spectrum. In model A, we considered stochastic acceleration by turbulence and solve the diffusion equation in momentum space. In models B and C, we do not specify the acceleration mechanism and assumed an injection term with a power-law and an exponential cutoff. Using such proton spectra, we have numerically calculated the neutrino spectra, taking account of the relevant cooling processes and the decay spectra. Since $p p$ inelastic collisions provide the main channel for high-energy neutrino production, the neutrino spectrum follows the proton spectrum. Close to the cutoff energy, $\varepsilon_{\nu} \sim 100 \mathrm{TeV}$, the photomeson production is as efficient as $p p$ interactions, leading to a comparable contribution to the neutrino flux. With a few to 10 LLAGNs stacked, a 10-year operation of IceCube-Gen2 will enable us to detect a few to several neutrinos from LLAGNs, otherwise they will put meaningful constraints on the parameter space. On the other hand, the cascade emission is difficult to detect with Fermi or CTA. Bright objects have a lower $\gamma \gamma$ cutoff energy, while objects with 
a higher value of the cutoff energy are too dim to produce a detectable signal.

AGN coronae and RIAFs are thought to be promising sites of particle acceleration, and accompanying papers suggest the AGN cores as the main origin of the mysterious $10-100 \mathrm{TeV}$ component in the diffuse neutrino flux observed in IceCube [87]. The model predicts that both Seyfert galaxies and LLAGNs are promising sources of high-energy neutrinos and $\mathrm{MeV}$ gamma rays. Our studies suggest the relevance of multi-messenger searches for
LLAGNs whether the $10-100 \mathrm{TeV}$ neutrinos mainly come from Seyfert galaxies or LLAGNs.

\section{ACKNOWLEDGMENTS}

This work is supported in part by JSPS Oversea Research Fellowship, JSPS Postdoctral Fellowship, the IGC postdoctoral fellowship program (S.S.K.), the Alfred P. Sloan Foundation, NSF Grant No. PHY-1620777 (K.M.), and the Eberly Foundation (P.M.).
[1] M. G. Aartsen, R. Abbasi, Y. Abdou, M. Ackermann, J. Adams, J. A. Aguilar, M. Ahlers, D. Altmann, J. Auffenberg, X. Bai, and et al., Physical Review Letters 111, 021103 (2013), arXiv:1304.5356 [astro-ph.HE].

[2] IceCube Collaboration, Science 342, 1242856 (2013) arXiv:1311.5238 [astro-ph.HE]

[3] M. G. Aartsen, M. Ackermann, J. Adams, J. A. Aguilar, M. Ahlers, M. Ahrens, D. Altmann, T. Anderson, C. Arguelles, T. C. Arlen, and et al., Physical Review Letters 113, 101101 (2014), arXiv:1405.5303 [astro-ph.HE]

[4] M. G. Aartsen, M. Ackermann, J. Adams, J. A. Aguilar, M. Ahlers, M. Ahrens, D. Altmann, T. Anderson, C. Arguelles, T. C. Arlen, and et al., Phys. Rev. D 91, 022001 (2015) arXiv:1410.1749 [astro-ph.HE]

[5] M. G. Aartsen, K. Abraham, M. Ackermann, J. Adams, J. A. Aguilar, M. Ahlers, M. Ahrens, D. Altmann, T. Anderson, M. Archinger, and et al., ApJ 809, 98 (2015), arXiv:1507.03991 [astro-ph.HE].

[6] M. Ahlers and F. Halzen, Progress of Theoretical and Experimental Physics 2017,
E. Waxman and J. Physical Review Letters 78, 2292 (1997) astro-ph/9701231

[8] P. Mészáros and E. Waxman, Physical Review Letters 87, 171102 (2001) astro-ph/0103275.

[9] C. D. Dermer and A. Atoyan, Phys. Rev. Lett. 91, 071102 (2003), arXiv:astro-ph/0301030 [astro-ph]

[10] D. Guetta, D. Hooper, J. Alvarez-Mun Iz, F. Halzen, and E. Reuveni, Astroparticle Physics 20, 429 (2004) astro-ph/0302524.

[11] K. Murase and S. Nagataki, Phys. Rev. D 73, 063002 (2006), astro-ph/0512275.

[12] M. G. Aartsen, K. Abraham, M. Ackermann, J. Adams, J. A. Aguilar, M. Ahlers, M. Ahrens, D. Altmann, T. Anderson, I. Ansseau, and et al., ApJ 824, 115 (2016), arXiv:1601.06484 [astro-ph.HE].

[13] M. G. Aartsen, M. Ackermann, J. Adams, J. A. Aguilar, M. Ahlers, M. Ahrens, I. A. Samarai, D. Altmann, K. Andeen, T. Anderson, and et al., ApJ 843, 112 (2017), arXiv:1702.06868 [astro-ph.HE],

[14] E. Waxman and J. N. Bahcall, ApJ 541, 707 (2000), hep-ph/9909286
[15] K. Murase and S. Nagataki, Physical Review Letters 97, 051101 (2006) astro-ph/0604437.

[16] K. Murase, Phys. Rev. D 76, 123001 (2007) arXiv:0707.1140.

[17] S. S. Kimura, K. Murase, P. Mészáros, and K. Kiuchi, ApJ 848, L4 (2017), arXiv:1708.07075 [astro-ph.HE]

[18] K. Murase, K. Ioka, S. Nagataki, and T. Nakamura, ApJ 651, L5 (2006), astro-ph/0607104.

[19] N. Senno, K. Murase, and P. Mészáros, Phys. Rev. D 93, 083003 (2016), arXiv:1512.08513 [astro-ph.HE].

[20] B. T. Zhang, K. Murase, S. S. Kimura, S. Horiuchi, and P. Mészáros, Phys. Rev. D 97, 083010 (2018), arXiv:1712.09984 [astro-ph.HE].

[21] D. Boncioli, D. Biehl, and W. Winter, ApJ 872, 110 (2019), arXiv:1808.07481 [astro-ph.HE].

[22] B. T. Zhang and K. Murase, arXiv e-prints , arXiv:1812.10289 (2018), arXiv:1812.10289 [astro-ph.HE],

[23] S. Razzaque, P. Mészáros, and E. Waxman, $\quad$ Phys. Rev. D 68, 083001 (2003)

105 a(2)1V:)istro-ph/0303505 [astro-ph]

[24] S. Ando and J. F. Beacom, Phys. Rev. Lett. 95, 061103 (2005), arXiv:astro-ph/0502521 [astro-ph]

[25] S. Horiuchi and S. Ando, Phys. Rev. D 77, 063007 (2008), arXiv:0711.2580

[26] K. Murase and K. Ioka, Physical Review Letters 111, 121102 (2013) arXiv:1306.2274 [astro-ph.HE]

[27] H.-N. He, A. Kusenko, S. Nagataki, Y.-Z. Fan, and D.-M. Wei, ApJ 856, 119 (2018), arXiv:1803.07478 [astro-ph.HE],

[28] P. B. Denton and I. Tamborra, ApJ 855, 37 (2018) arXiv:1711.00470 [astro-ph.HE],

[29] S. S. Kimura, K. Murase, I. Bartos, K. Ioka, I. S. Heng, and P. Mészáros, Phys. Rev. D 98, 043020 (2018) arXiv:1805.11613 [astro-ph.HE]

[30] K. Mannheim, T. Stanev, and P. L. Biermann, A\&A 260, L1 (1992).

[31] F. Halzen and E. Zas, ApJ 488, 669 (1997) arXiv:astro-ph/9702193 [astro-ph]

[32] A. Atoyan and C. D. Dermer, Physical Review Letters 87, 221102 (2001), astro-ph/0108053.

[33] IceCube-Collaboration, Fermi-LAT, MAGIC, AG- 
ILE, ASAS-SN, HAWC, H.E.S.S, INTEGRAL, Kanata, Kiso, Kapteyn, L. Telescope, Subaru, Swift/NuSTAR, VERITAS, V.-. teams, et al. (IceCube Collaboration), Science 361, 146 (2018), arXiv:1807.xxxxx [astro-ph.HE]

[34] M. G. Aartsen et al. (IceCube), Astropart. Phys. 92, 30 (2017) arXiv:1612.06028 [astro-ph.HE].

[35] A. Keivani, K. Murase, M. Petropoulou, D. B. Fox, S. B. Cenko, S. Chaty, A. Coleiro, J. J. DeLaunay, S. Dimitrakoudis, P. A. Evans, J. A. Kennea, F. E. Marshall, A. Mastichiadis, J. P. Osborne, M. Santand er, A. Tohuvavohu, and C. F. Turley, ApJ 864, 84 (2018) arXiv:1807.04537 [astro-ph.HE]

[36] K. Murase, F. Oikonomou, and M. Petropoulou, ApJ 865, 124 (2018), arXiv:1807.04748 [astro-ph.HE].

[37] R.-Y. Liu, K. Wang, R. Xue, A. M. Taylor, X.-Y. Wang, Z. Li, and H. Yan, arXiv e-prints , arXiv:1807.05113 (2018), arXiv:1807.05113 [astro-ph.HE]

[38] M. Cerruti, A. Zech, C. Boisson, G. Emery, S. Inoue, and J. P. Lenain, MNRAS 483, L12 (2019), arXiv:1807.04335 [astro-ph.HE]

[39] S. Gao, A. Fedynitch, W. Winter, and M. Pohl, Nature Astronomy 3, 88 (2019), arXiv:1807.04275 [astro-ph.HE]

[40] IceCube-Collaboration (IceCube Collaboration), Science 361, 147 (2018), arXiv:1807.xxxxx [astro-ph.HE]

[41] S. Garrappa et al. (Fermi-LAT, ASAS-SN, IceCube), (2019), arXiv:1901.10806 [astro-ph.HE]

[42] A. Reimer, M. Boettcher, and S. Buson, arXiv e-prints , arXiv:1812.05654 (2018), arXiv:1812.05654 [astro-ph.HE].

[43] X. Rodrigues, S. Gao, A. Fedynitch, A. Palladino, and W. Winter, arXiv e-prints , arXiv:1812.05939 (2018), arXiv:1812.05939 [astro-ph.HE],

[44] K. Wang, R.-Y. Liu, Z. Li, X.-Y. Wang, and Z.-G. Dai, arXiv e-prints , arXiv:1809.00601 (2018), arXiv:1809.00601 [astro-ph.HE],

[45] M. G. Aartsen et al. (IceCube), Astrophys. J. 835, 45 (2017) arXiv:1611.03874 [astro-ph.HE]

[46] A. Neronov, D. V. Semikoz, and K. Ptitsyna, $\quad$ Astron. Astrophys. 603, A135 (2017) arXiv:1611.06338 [astro-ph.HE]

[47] C. Yuan, K. Murase, and P. Mészáros, arXiv e-prints , arXiv:1904.06371 (2019), arXiv:1904.06371 [astro-ph.HE].

[48] D. Hooper, T. Linden, and A. Vieregg, J. Cosmology Astropart. Phys. 2019, 012 (2019) arXiv:1810.02823 [astro-ph.HE].

[49] K. Murase and E. Waxman, Phys. Rev. D94, 103006 (2016), arXiv:1607.01601 [astro-ph.HE].

[50] M. Ackermann et al. (FermiLAT), Astrophys. J. 799, 86 (2015) arXiv:1410.3696 [astro-ph.HE],

[51] K. Murase, M. Ahlers, and B. C. Lacki, $\quad$ Phys. Rev. D 88, 121301 (2013) arXiv:1306.3417 [astro-ph.HE],

[52] M. G. Aartsen et al. (IceCube), Phys. Rev. Lett. 115, 081102 (2015), arXiv:1507.04005 [astro-ph.HE].

[53] M. G. Aartsen et al. (IceCube Collaboration), (2017), arXiv:1710.01179 [astro-ph.HE].
[54] A. Palladino and W. Winter, A\&A 615, A168 (2018), arXiv:1801.07277 [astro-ph.HE],

[55] K. Murase, D. Guetta, and M. Ahlers, Phys. Rev. Lett. 116, 071101 (2016), arXiv:1509.00805 [astro-ph.HE]

[56] A. Loeb and E. Waxman, J. Cosmology Astropart. Phys. 5, 003 (2006) astro-ph/0601695.

[57] H.-N. He, T. Wang, Y.-Z. Fan, S.-M. Liu, and D.-M. Wei, Phys. Rev. D 87, 063011 (2013) arXiv:1303.1253 [astro-ph.HE]

[58] L. A. Anchordoqui, T. C. Paul, L. H. M. da Silva, D. F. Torres, and B. J. Vlcek, $\quad$ Phys. Rev. D 89, 127304 (2014), arXiv:1405.7648 [astro-ph.HE]

[59] R.-Y. Liu, X.-Y. Wang, S. Inoue, R. Crocker, and F. Aharonian, Phys. Rev. D 89, 083004 (2014), arXiv:1310.1263 [astro-ph.HE]

[60] I. Tamborra, S. Ando, and K. Murase, J. Cosmology Astropart. Phys. 9, 043 (2014) arXiv:1404.1189 [astro-ph.HE]

[61] N. Senno, P. Mészáros, K. Murase, P. Baerwald, and M. J. Rees, ArXiv e-prints (2015), arXiv:1501.04934 [astro-ph.HE].

[62] D. Xiao, P. Mészáros, K. Murase, and Z.-G. Dai, ApJ 826, 133 (2016), arXiv:1604.08131 [astro-ph.HE].

[63] K. Bechtol, M. Ahlers, M. Di Mauro, M. Ajello, and J. Vandenbroucke, ApJ 836, 47 (2017) arXiv:1511.00688 [astro-ph.HE],

[64] T. Sudoh, T. Totani, and N. Kawanaka, Publications of the Astronomical Society of Japan 70, 49 (2018) arXiv:1801.09683 [astro-ph.HE].

[65] K. Murase, S. Inoue, and S. Nagataki, ApJ 689, L105 (2008), arXiv:0805.0104.

[66] K. Kotera, D. Allard, K. Murase, J. Aoi, Y. Dubois, T. Pierog, and S. Nagataki, ApJ 707, 370 (2009), arXiv:0907.2433 [astro-ph.HE]

[67] F. Zandanel, I. Tamborra, S. Gabici, and S. Ando, A\&A 578, A32 (2015), arXiv:1410.8697 [astro-ph.HE],

[68] K. Fang and A. V. Olinto, ApJ 828, 37 (2016), arXiv:1607.00380 [astro-ph.HE],

[69] K. Fang and K. Murase, Nature Physics 14, 396 (2018) arXiv:1704.00015 [astro-ph.HE]

[70] J. Becker Tjus, B. Eichmann, F. Halzen, A. Kheirandish, and S. M. Saba, Phys. Rev. D 89, 123005 (2014), arXiv:1406.0506 [astro-ph.HE]

[71] D. Hooper, Journal of Cosmology and Astro-Particle Physics 2016, arXiv:1605.06504 [astro-ph.HE]

[72] V. S. Berezinskii and V. L. Ginzburg, MNRAS 194, 3 (1981).

[73] D. Kazanas and D. C. Ellison, ApJ 304, 178 (1986).

[74] A. A. Zdziarski, ApJ 305, 45 (1986)

[75] M. C. Begelman, B. Rudak, and M. Sikora, ApJ 362, 38 (1990)

[76] F. W. Stecker, C. Done, M. H. Salamon, and P. Sommers, Physical Review Letters 66, 2697 (1991)

[77] W. Bednarek and Protheroe, $\quad$ MNRAS 302, 373 (1999), arXiv:astro-ph/9802288 [astro-ph]

[78] J. Alvarez-Muñiz and P. Mészáros, Phys. Rev. D 70, 123001 (2004), astro-ph/0409034

[79] N. I. Shakura and R. A. Sunyaev, A\&A 24, 337 (1973).

[80] M. A. Malkan, ApJ 268, 582 (1983).

[81] B. Czerny and M. Elvis, ApJ 321, 305 (1987). 
[82] A. Marconi, G. Risaliti, R. Gilli, L. K. Hunt, R. Maiolino, and M. Salvati, MNRAS 351, 169 (2004) astro-ph/0311619

[83] P. F. Hopkins, G. T. Richards, and L. Hernquist, $\quad$ ApJ 654, 731 (2007) arXiv:astro-ph/0605678 [astro-ph]

[84] E. Lusso, A. Comastri, B. D. Simmons, M. Mignoli, G. Zamorani, C. Vignali, M. Brusa, F. Shankar, D. Lutz, J. R. Trump, R. Maiolino, R. Gilli, M. Bolzonella, S. Puccetti, M. Salvato, C. D. Impey, F. Civano, M. Elvis, V. Mainieri, J. D. Silverman, A. M. Koekemoer, A. Bongiorno, A. Merloni, S. Berta, E. Le Floc'h, B. Magnelli, F. Pozzi, and L. Riguccini, MNRAS 425, 623 (2012), arXiv:1206.2642 [astro-ph.CO]

[85] O. Kalashev, D. Semikoz, and I. Tkachev, Soviet Journal of Experimental and Theoretical Physics arXiv:1410.8124 [astro-ph.HE].

[86] Y. Inoue, D. Khangulyan, S. Inoue, and A. Doi, arXiv e-prints , arXiv:1904.00554 (2019), arXiv:1904.00554 [astro-ph.HE]

[87] K. Murase, S. S. Kimura, and P. Meszaros, arXiv e-prints , arXiv:1904.04226 (2019), arXiv:1904.04226 [astro-ph.HE]

[88] L. C. Ho, ARA\&A 46, 475 (2008), arXiv:0803.2268

[89] R. Narayan and I. Yi, ApJ 428, L13 (1994), astro-ph/9403052

[90] F. Yuan and R. Narayan, ARA\&A 52, 529 (2014). arXiv:1401.0586 [astro-ph.HE].

[91] S. S. Kimura, K. Murase, and K. Toma, ApJ 806, 159 (2015), arXiv:1411.3588 [astro-ph.HE]

[92] B. Khiali and E. M. de Gouveia Dal Pino, MNRAS 455, 838 (2016) arXiv:1506.01063 [astro-ph.HE],

[93] C. Righi, F. Tavecchio, and S. Inoue, MNRAS 483, L127 (2019), arXiv:1807.10506 [astro-ph.HE]

[94] M. Machida and R. Matsumoto, ApJ 585, 429 (2003), astro-ph/0211240.

[95] J. C. McKinney, MNRAS 368, 1561 (2006) astro-ph/0603045

[96] K. Ohsuga and S. Mineshige, ApJ 736, 2 (2011), arXiv:1105.5474 [astro-ph.HE].

[97] R. Narayan, A. SA dowski, R. F. Penna, and A. K. Kulkarni, MNRAS 426, 3241 (2012) arXiv:1206.1213 [astro-ph.HE].

[98] R. F. Penna, A. Sadowski, A. K. Kulkarni, and R. Narayan, MNRAS 428, 2255 (2013) arXiv:1211.0526 [astro-ph.HE]

[99] S. S. Kimura, K. Tomida, and K. Murase, MNRAS 485, 163 (2019) arXiv:1812.03901 [astro-ph.HE],

[100] S. M. Ressler, A. Tchekhovskoy, E. Quataert, and C. F. Gammie, MNRAS 467, 3604 (2017) arXiv:1611.09365 [astro-ph.HE]

[101] A. Chael, M. Rowan, R. Narayan, M. Johnson, and L. Sironi, MNRAS 478, 5209 (2018) arXiv:1804.06416 [astro-ph.HE]

[102] D. Ball, L. Sironi, and F. Özel, ApJ 862, 80 (2018), arXiv:1803.05556 [astro-ph.HE]

[103] R. G. Martin, C. J. Nixon, J. E. Pringle, and M. Livio, arXiv e-prints , arXiv:1901.01580 (2019), arXiv:1901.01580 [astro-ph.HE]
[104] F.-G. Xie and F. Yuan, MNRAS 427, 1580 (2012) arXiv:1207.3113 [astro-ph.HE]

[105] R. Mahadevan and E. Quataert, ApJ 490, 605 (1997), astro-ph/9705067.

[106] S. Kato, J. Fukue, and S. Mineshige, Black-Hole Accretion Disks - Towards a New Paradigm -, 549 pages, including 12 Chapters, 9 Appendices, ISBN 9784-87698-740-5, Kyoto University Press (Kyoto, Japan), 2008. (Kyoto University Press, 2008).

[107] R. Narayan and I. Yi, ApJ 452, 710 (1995) astro-ph/9411059.

[108] M. A. Abramowicz, X. Chen, S. Kato, J.-P. Lasota, and O. Regev, ApJ 438, L37 (1995), astro-ph/9409018

[109] R. Mahadevan, astro-ph/9609107.

[110] E. Quataert and A. Gruzinov, ApJ 520, 248 (1999) 20, 541a(280d-5) ) $/ / 9803112$.

[111] P. Sharma, E. Quataert, G. W. Hammett, and J. M. Stone, ApJ 667, 714 (2007), astro-ph/0703572.

[112] M. A. Riquelme, E. Quataert, P. Sharma, and A. Spitkovsky, ApJ 755, 50 (2012) arXiv:1201.6407 [astro-ph.HE]

[113] M. W. Kunz, A. A. Schekochihin, and J. M. Stone, Physical Review Letters 112, 205003 (2014), arXiv:1402.0010 [astro-ph.HE]

[114] M. Hoshino, arXiv:1306.6720 [astro-ph.HE]

ApJ 773, 118 (2013)

[115] M. Hoshino, Physical Review Letters 114, 061101 (2015) arXiv:1502.02452 [astro-ph.HE].

[116] L. Sironi and R. Narayan, ApJ 800, 88 (2015) arXiv:1411.5685 [astro-ph.HE]

[117] L. Sironi, arXiv:1411.6014 [astro-ph.HE]

ApJ 800, 89 (2015)

[118] V. Zhdankin, D. A. Uzdensky, G. R. Werner, and M. C. Begelman, arXiv e-prints , arXiv:1809.01966 (2018), arXiv:1809.01966 [astro-ph.HE].

[119] J.-M. Wang, K.-Y. Watarai, and S. Mineshige, ApJ 607, L107 (2004) arXiv:astro-ph/0407160 [astro-ph]

[120] Z. Liu, A. Merloni, A. Georgakakis, M.-L. Menzel, J. Buchner, K. Nandra, M. Salvato, Y. Shen, M. Brusa, and A. Streblyanska, MNRAS 459, 1602 (2016) arXiv:1605.00207 [astro-ph.HE].

[121] P. Saikia, E. Körding, D. L. Coppejans, H. Falcke, D. Williams, R. D. Baldi, I. Mchardy, and R. Beswick, A\&A 616, A152 (2018) arXiv:1805.06696 [astro-ph.HE]

[122] M. Eracleous, J. A. Hwang, and H. M. L. G. Flohic, ApJS 187, 135 (2010), arXiv:1001.2924 [astro-ph.GA],

[123] M. Gu and X. Cao, MNRAS 399, 349 (2009) arXiv:0906.3560 [astro-ph.GA]

[124] R. She, L. C. Ho, H. Feng, and C. Cui, ApJ 859, 152 (2018), arXiv:1804.07482 [astro-ph.HE].

[125] G. Younes, A. Ptak, L. C. Ho, F.-G. Xie, Y. Terasima, F. Yuan, D. Huppenkothen, and M. Yukita, ApJ 870, 73 (2019), arXiv:1811.10657 [astro-ph.GA]

[126] A. De Angelis, V. Tatischeff, M. Tavani, U. Oberlack, I. Grenier, L. Hanlon, R. Walter, A. Argan, P. von Ballmoos, and A. Bulgarelli, $\quad$ Experimental Astronomy 44, 25 (2017). arXiv:1611.02232 [astro-ph.HE].

[127] A. Moiseev and Amego Team, International Cosmic Ray Conference 301, 798 (2017).

[128] T. Aramaki, P. O. H. Adrian, G. Karagiorgi, 
and H. Odaka, Astroparticle Physics 114, 107 (2020), arXiv:1901.03430 [astro-ph.HE]

[129] S. Zenitani and M. Hoshino, ApJ 562, L63 (2001) arXiv:1402.7139 [astro-ph.HE]

[130] G. Kowal, E. M. de Gouveia Dal Pino, and A. Lazarian, Physical Review Letters 108, 241102 (2012) arXiv:1202.5256 [astro-ph.HE]

[131] L. Sironi and A. Spitkovsky, ApJ 783, L21 (2014) arXiv:1401.5471 [astro-ph.HE]

[132] M. W. Kunz, J. M. Stone, and E. Quataert, Physical Review Letters 117, 235101 (2016), arXiv:1608.07911 [astro-ph.HE].

[133] S. S. Kimura, K. Toma, T. K. Suzuki, and S.-i. Inutsuka, ApJ 822, 88 (2016) arXiv:1602.07773 [astro-ph.HE].

[134] L. Comisso and L. Sironi, Phys. Rev. Lett. 121, 255101 (2018) arXiv:1809.01168 [astro-ph.HE].

[135] K. Wong, V. Zhdankin, D. A. Uzdensky, G. R. Werner, and M. C. Begelman, arXiv e-prints , arXiv:1901.03439 (2019), arXiv:1901.03439 [astro-ph.HE]

[136] A. Y. Chen, Y. Yuan, and H. Yang, ApJ 863, L31 (2018), arXiv:1805.11039 [astro-ph.HE]

[137] A. Levinson and B. Cerutti, A\&A 616, A184 (2018), arXiv:1803.04427 [astro-ph.HE]

[138] F. Takahara and M. Kusunose, Progress of Theoretical Physics 73, 1390 (1985).

[139] S. S. Kimura, K. Toma, and F. Takahara, ApJ 791, 100 (2014), arXiv:1407.0115 [astro-ph.HE].

[140] J. E. Pringle, ARA\&A 19, 137 (1981).

[141] M. A. Abramowicz, B. Czerny, J. P. Lasota, and E. Szuszkiewicz, ApJ 332, 646 (1988).

[142] R. Blandford and D. Eichler, Phys. Rep. 154, 1 (1987)

[143] V. Petrosian, Space Sci. Rev. 173, 535 (2012), arXiv:1205.2136 [astro-ph.HE]

[144] C. D. Dermer, J. A. Miller, and H. Li, ApJ 456, 106 (1996) astro-ph/9508069

[145] Ł. Stawarz and V. Petrosian, ApJ 681, 1725 (2008) arXiv:0803.0989.

[146] J. Kakuwa, $\quad$ ApJ 816, 24 (2016) \begin{tabular}{|llll}
$\operatorname{arXiv}: 1511.07738$ [astro-ph.HE] & \\
J. S. Chang and & G. Cooper,
\end{tabular} Journal of Computational Physics 6, 1 (1970)

[148] B. T. Park and V. Petrosian, The Astrophysical Journal Supplement Series 103, 255 (1996)
[149] C. D. Dermer and G. Menon, High Energy Radiation from Black Holes: Gamma Rays, Cosmic Rays, and Neutrinos (2009).

[150] J. Giacalone and J. R. Jokipii, ApJ 520, 204 (1999)

[151] F. Casse, M. Lemoine, and G. Pelletier, Phys. Rev. D 65, 023002 (2002), astro-ph/0109223

[152] E. Kafexhiu, F. Aharonian, A. M. Taylor, and G. S. Vila, Phys. Rev. D 90, 123014 (2014), arXiv:1406.7369 [astro-ph.HE]

[153] S. Stepney and P. W. Guilbert, MNRAS 204, 1269 (1983)

[154] M. J. Chodorowski, A. A. Zdziarski, and M. Sikora, ApJ 400, 181 (1992)

[155] P. A. Becker, T. Le, and C. D. Dermer, ApJ 647, 539 (2006), astro-ph/0604504.

[156] S. R. Kelner, F. A. Aharonian, and V. V. Bugayov, Phys. Rev. D 74, 034018 (2006), astro-ph/0606058

[157] R. Laha, J. F. Beacom, B. Dasgupta, S. Horiuchi, and K. Murase, Phys. Rev. D88, 043009 (2013) arXiv:1306.2309 [astro-ph.HE]

[158] M. G. Aartsen et al. (IceCube), Astrophys. J. 796, 109 (2014) arXiv:1406.6757 [astro-ph.HE]

[159] K. Murase, $\quad$ Phys. Rev. D 97, 081301 (2018) arXiv:1705.04750 [astro-ph.HE]

[160] K. Murase, A. Franckowiak, K. Maeda, R. Margutti, and J. F. Beacom, ApJ 874, 80 (2019) arXiv:1807.01460 [astro-ph.HE].

[161] P. S. Coppi and R. D. Blandford, MNRAS 245, 453 (1990).

[162] R. Genzel, F. Eisenhauer, and S. Gillessen, Reviews of Modern Physics 82, 3121 (2010) arXiv:1006.0064 [astro-ph.GA]

[163] F. K. Baganoff, Y. Maeda, M. Morris, M. W. Bautz, W. N. Brandt, W. Cui, J. P. Doty, E. D. Feigelson, G. P. Garmire, S. H. Pravdo, G. R. Ricker, and L. K. Townsley, ApJ 591, 891 (2003), arXiv:astro-ph/0102151 [astro-ph]

[164] D. Porquet, P. Predehl, B. Aschenbach, N. Grosso, A. Goldwurm, P. Goldoni, R. S. Warwick, and A. Decourchelle, A\&A 407, L17 (2003) arXiv:astro-ph/0307110 [astro-ph]

[165] J. C. Rodríguez-Ramírez, E. M. de Gouveia Dal Pino, and R. Alves Batista, ApJ 879, 6 (2019), arXiv:1904.05765 [astro-ph.HE] 